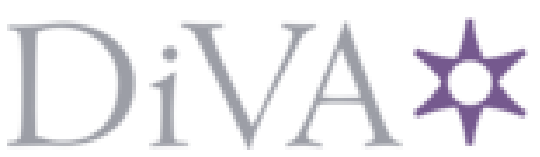

http://www.diva-portal.org

[This is not an article, chapter, of conference paper!] 


\title{
Degrees of Freedom of Two-Hop MISO Broadcast Networks with Mixed CSIT
}

\author{
Zhao Wang, Ming Xiao, Chao Wang, and Mikael Skoglund
}

\begin{abstract}
A downlink two-hop MISO broadcast network is considered, with a two-antenna source communicating to 2 single-antenna destinations, via multiple single-antenna relays in between. The sum degrees of freedom (DOF) of the network with mixed channel state information at the transmitter (CSIT) is investigated. The mixed CSIT consists of accurate delayed CSIT and inaccurate instantaneous CSIT, and its availability is limited within each hop, i.e. the source is oblivious to the channels of the second hop. Given a transmission power $P$ and a real value $\alpha \in[0,1]$, if the variance of the error for instantaneous CSIT decreases as $O\left(P^{-\alpha}\right)$, it is shown that the sum optimal DOF of the considered network is $d=\frac{4+2 \alpha}{3}$ when there exist at least 3 intermediate relays. The result can be extended to the MIMO and multiple-hop cases. The proposed achievable schemes essentially combine the concept of retrospective interference alignment based on delayed CSIT and linear beamforming based on inaccurate instantaneous CSIT into an integrated form. Our results show that, in multi-hop MISO broadcast networks, delayed CSIT and inaccurate instantaneous CSIT can be exploited simultaneously to benefit network DOF.
\end{abstract}

Index Terms-Degrees of freedom, mixed CSIT, multi-hop system, interference alignment/neutralization.

\section{INTRODUCTION}

Channel state information at transmitter (CSIT) plays an important role in providing degrees of freedom (DOF) gains in wireless networks. In practical systems, channel state information (CSI) is normally estimated by the receivers from pilot symbols. Then CSIT is obtained via feedback. Due to the fading nature of wireless channels, perfect CSIT is hard to guarantee. Hence in practice various forms of imperfect CSIT may be attained instead, resulting in different DOF performance.

Considering a downlink broadcast channel (BC), a 2antenna source intends to communicate to two single-antenna destinations through identically and independently distributed (i.i.d.) fading channels. When the channel changes slowly, it is possible to attain instantaneous CSIT with relatively high accuracy. In this case, the CSIT is assumed to be perfect and the system has DOF 2, which can be achieved by dirty-paper coding [1] or linear beamforming (LBF) [2]. In general, nonnegligible CSIT estimation errors lead to a reduced DOF. For example, if the variance of the estimation error decays as $O\left(P^{-\alpha}\right)$ for some constant $\alpha \in[0,1]$ and transmission power $P, 2 \alpha$ DOF (i.e., only a fraction of the DOF when perfect CSIT is available) can be achieved by LBF [3]. If the channel

Z. Wang, M. Xiao, and M. Skoglund are with the Communication Theory Lab, School of Electrical Engineering, Royal Institute of Technology (KTH), Stockholm, Sweden (E-mail:\{zhaowang, mingx, skoglund $\} @$ kth.se). C. Wang is with the School of Electronics and Information Engineering, Tongji University, Shanghai, China (email: chaowang@tongji.edu.cn). varies rapidly, feedback delay may be comparable to channel coherence time. The extreme scenario in which transmitterside channel knowledge is completely outdated is modeled as delayed CSIT in [4]. With long feedback block length, it is easier for delayed CSIT to be sufficiently accurate, for instance, by high resolution CSI quantization [5]-[7]. Then, with accurate delayed CSIT, the sum DOF of the considered two-user $\mathrm{BC}$ is $\frac{4}{3}$, which can be achieved by a retrospective interference alignment (RIA) scheme [4] (we name the scheme following [8]). With the DOF higher than 1 (the sum DOF when no CSIT is available for isotropic fading BC [9]), it reveals that even completely outdated CSIT can improve the DOF of some wireless networks.

A further step towards the study of exploiting CSIT in wireless networks has been taken recently by [10] through the model of mixed CSIT, where both delayed CSIT, provided by a significantly delayed feedback channel, and instantaneous CSIT, attained via predictions based on the outdated CSIT or observation of reciprocal reverse channels, can be available. Recent works exhibit that wireless networks with both forms of CSIT may have even higher DOF than those with only one. For instance, it has been shown that when the variance of the estimation error for instantaneous CSIT decays as $O\left(P^{-\alpha}\right)$, the two-user BC has sum DOF $\frac{4+2 \alpha}{3} \in\left[\frac{4}{3}, 2\right]$ [11] [12]. Clearly, situations in which only accurate delayed CSIT (i.e. no knowledge of instantaneous CSIT) or perfect CSIT (i.e. the instantaneous CSIT is sufficiently accurate) are included as special cases and represented by $\alpha=0$ and $\alpha=1$ respectively. Therefore, the study on mixed CSIT provides a more comprehensive understanding towards the impact of CSIT on system DOF performance.

In the aforementioned system, if the source signals cannot directly reach the destinations due to, for instance, large distance between them, intermediate relays can be deployed to conduct communication. Finding the DOF in even small multi-hop systems is more challenging than in conventional single-hop networks. This is due to the fact that introducing relays potentially brings additional issues such as extra interuser interference, and extra load of channel estimation and signal processing. Furthermore, the optimal relay operation is far from being well understood. Inspired by the concepts of interference alignment (IA) [13]-[15] and/or interference neutralization [16]-[20], several pioneering works [21]-[25] have recently looked at the DOF in relay networks. These investigations are in general based on the condition that perfect CSI of the entire network is available at all terminals. Such an assumption is hard to realize, especially in layered multihop networks where the terminals may not be physically 
connected.

To study the impact of imperfect CSIT on DOF in multihop networks, we consider a two-hop MISO broadcast network with a 2-antenna source and 2 single-antenna destinations [26]. The source and the destinations are physically unconnected such that information delivery is assisted by multiple singleantenna relays. Assume that CSIT is attained through feedback signals from receivers. Regarding the transmission range of feedback signals, two scenarios can be considered.

CSIT via global feedback: Feedback signals from any receiver can reach the source. In other words, the source has the channel knowledge regarding both hops. Considering isotropic fading, and let $\overline{\mathbf{F}}$ and $\overline{\mathbf{G}}$ denote the channel matrices of the first and second hops available at transmitter side via feedback, respectively. Based on $\overline{\mathbf{F}}$ and $\overline{\mathbf{G}}$, the source can design its transmission strategy by treating the two-hop network as an equivalent single-hop BC, whose channel matrix is represented by $\overline{\mathbf{H}}=\overline{\mathbf{F}} \cdot \overline{\mathbf{G}}$ (omitting the amplification factor at relays). Thus the aforementioned DOF results for the single-hop BC are applicable here. Clearly, attaining global CSI feedback requires feedback links between the physically unconnected destinations and source. This may not be applicable in many systems.

CSIT via local feedback: Feedback signals from any receiver can reach only the terminals in the previous layer. Specifically, the source attains $\overline{\mathbf{F}}$ but is oblivious to $\overline{\mathbf{G}}$, which is available at the relays. Due to the CSI limitation, a straightforward cascade approach sees the network as a concatenation of a single hop BC (the first hop) and an X channel (the second hop) [15]. By this approach, one may conjecture that the system sum DOF is limited by that of the $\mathrm{X}$ channel, and is thus smaller than the DOF of a single-hop BC. However, we can apply transmission strategies similar to those proposed in [16], [19], [20], [27] to show its suboptimality when either perfect CSIT or only delayed CSIT can be obtained, and the multi-hop broadcast network has the same DOF as the single-hop BC. Now, it is natural to ask what will happen if mixed CSIT is attainable. We aim to answer this question in this paper.

The main contributions of this paper are as follows. We first establish the sum DOF of the two-hop MISO broadcast network with mixed CSIT when at least 3 relays are deployed. Specifically, we show that when the variance of the instantaneous CSIT estimation error decays as $O\left(P^{-\alpha}\right)$, where $\alpha \in[0,1]$, the network's sum DOF is $\frac{4+2 \alpha}{3}$. This result remains true even when the communication between the source and its destinations has to be conducted by multiple layers of relays. Noting that $\frac{4+2 \alpha}{3}$ is the sum DOF of the corresponding single-hop BC, our results show that in the considered setup delivering signals through multiple layers of distributed relay processing will not lead to degraded DOF. The analysis can be extended to multiple-antenna scenarios. When the source has $2 M$ antennas and the other terminals have $M$ antennas each, the system sum DOF is enhance to $\frac{(4+2 \alpha) M}{3}$. However, for the two-hop MISO network with only 2 relays, the DOF remains unsettled with mixed CSIT. It is worth noting that the proposed achievable schemes in all these cases essentially combine the concept of retrospective interference alignment based on

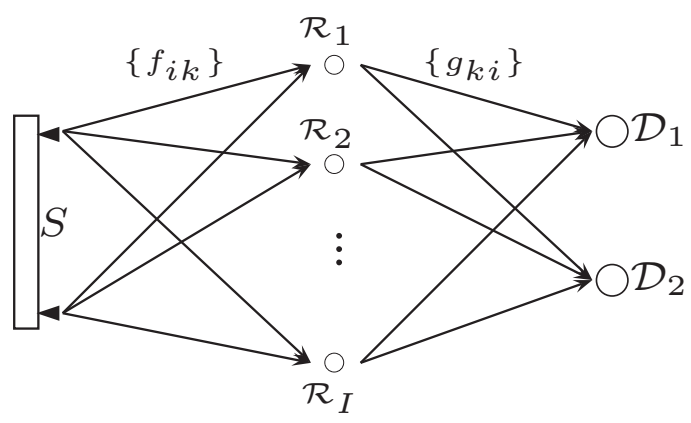

Fig. 1. System model: A $2 \times I \times 2$ MISO broadcast network. $f_{i k}$ represents the channel coefficient between the antenna $k$ of $\mathcal{S}$ and $\mathcal{R}_{i}$, and $g_{k i}$ represents the channel coefficient between $\mathcal{R}_{i}$ and $\mathcal{D}_{k}$, for $k \in\{1,2\}$ and $i \in\{1,2, \ldots, I\}$.

delayed CSIT and linear beamforming based on inaccurate instantaneous CSIT into an integrated form. Therefore, we can conclude that in multi-hop MISO broadcast networks, delayed CSIT and inaccurate instantaneous CSIT can be exploited simultaneously to benefit network DOF.

The rest of the paper is organized as follows. In Section II, we introduce our two-hop MISO broadcast network model. In Section III, we summarize the main result of the paper. The proof of the main result is provided in Section IV, with discussions presented in Section V. Finally, our conclusions are drawn in Section VI.

Notations: Superscripts ${ }^{T}$ and ${ }^{H}$ represent matrix transpose and Hermitian transpose, respectively. $\|A\|$ denotes the Euclidean norm of vector $A$. $|\mathbf{A}|$ represents the determinant of matrix A. $\mathbb{E}(\cdot)$ denotes the expectation operation. $g(x)=$ $O(f(x))$ if and only if there exists a positive real value $m$ and a real value $x_{0}$ such that $|g(x)| \leq m|f(x)|$ for all $x>x_{0}$. $g(x)=o(f(x))$ represents that $\lim _{x \rightarrow \infty} \frac{g(x)}{f(x)}=0 . A \perp B$ represents that vectors $A$ and $B$ are orthogonal. I denotes the identity matrix.

\section{SySTEM MODEL}

\section{A. Channel Model}

As shown in Fig. 1, we consider a 2-user 2-hop MISO broadcast network in which a source node $\mathcal{S}$ with 2 transmit antennas intends to send 2 independent messages to 2 singleantenna destinations $\mathcal{D}_{1}$ and $\mathcal{D}_{2}$, respectively. There is no direct link between $\mathcal{S}$ and $\mathcal{D}_{k}(k=1,2)$. $I(I \geq 2)$ singleantenna relays $\mathcal{R}_{i}(i \in\{1, \cdots, I\})$ are deployed between them to help the communication. We term this network a $2 \times I \times 2$ MISO broadcast network.

Let $P$ represent the long term average power of each node. The sum optimal DOF of the considered network is defined as $d=\lim _{P \rightarrow \infty} \frac{C_{\Sigma}(P)}{\log _{2} P}$ [28], where $C_{\Sigma}$ denotes the sum capacity.

$\left\{f_{i k}(b, t)\right\}$ and $\left\{g_{k i}(b, t)\right\}(k \in\{1,2\}, i \in\{1,2, \ldots, I\})$ represent the source-relay channel coefficients and the relaydestination channel coefficients at time slot $t$ in block $b$, respectively, for $t \in\{1,2, \ldots, T\}$ and $b \in \mathbb{Z}^{+}$, where $T \in \mathbb{Z}^{+}$represents the length of a block. For each pair of $(b, t), f_{i k}(b, t)$ and $g_{k i}(b, t)$ are assumed to be i.i.d., drawn from a continuous distribution. Their absolute values 
are bounded away from zero and infinity to avoid channel degenerations [14]. We consider a time-varying fading environment such that all channel coefficients remain constant within one time slot but change independently across different time slots. Let $F_{i}(b, t)=\left[f_{i 1}(b, t) f_{i 2}(b, t)\right]^{T}$ and $G_{k}(b, t)=$ $\left[g_{k 1}(b, t) g_{k 2}(b, t) \ldots g_{k I}(b, t)\right]^{T}$. The transmission signal of $\mathcal{S}$ at time slot $(b, t)$ is a $2 \times 1$ vector $X(b, t)$. Then, the received signal of relay $\mathcal{R}_{i}(i \in\{1,2, \cdots, I\})$ at time slot $(b, t)$ is

$$
y_{R_{i}}(b, t)=F_{i}^{T}(b, t) X(b, t)+z_{i}(b, t),
$$

where $z_{i}(b, t)$ is the unit-power additive white Gaussian noise (AWGN). We consider full-duplex relaying. At time slot $(b, t)$, the transmission signal of $\mathcal{R}_{i}$ is $x_{R_{i}}(b, t)$. Then at the second hop, the received signal of destination $\mathcal{D}_{k}(k \in\{1,2\})$ is

$$
y_{k}(b, t)=G_{k}^{T}(b, t) X_{R}(b, t)+n_{k}(b, t),
$$

where $X_{R}(b, t)=\left[\begin{array}{llll}x_{R_{1}}(b, t) & x_{R_{2}}(b, t) & \ldots & x_{R_{I}}(b, t)\end{array}\right]^{T}$ and $n_{k}(b, t)$ is unit-power AWGN.

\section{B. Mixed CSIT}

Throughout the paper, we assume perfect instantaneous CSI at receivers. However, two kinds of imperfections affect the CSIT, namely, inaccurate instantaneous CSIT and accurate delayed CSIT. We represent them as the mixed CSIT. We note that these two types of CSIT can be obtained by different approaches in practice. For instance, instantaneous CSIT can be attained by observing the reverse reciprocal channel, and delayed CSIT can be obtained by the high-resolution feedback.

1) Inaccurate instantaneous CSIT: At the transmitter side, the available CSI can only partially represent the instantaneous channel condition. We represent the channel coefficients as

$$
\begin{aligned}
& f_{i k}(b, t)=\hat{f}_{i k}(b, t)+\tilde{f}_{i k}(b, t), \\
& g_{k i}(b, t)=\hat{g}_{k i}(b, t)+\tilde{g}_{k i}(b, t),
\end{aligned}
$$

where $\hat{f}_{i k}(b, t)$ and $\hat{g}_{k i}(b, t)$ denote the estimated channel coefficients, and $\tilde{f}_{i k}(b, t), \tilde{g}_{k i}(b, t)$ denote the estimation error. We assume $\hat{f}_{i k}(b, t)$ and $\tilde{f}_{i k}(b, t)$ to be independent random variables with zero-mean and bounded finite moments. Their variances are $1-\delta^{2}$ and $\delta^{2}$, respectively $\left(0 \leq \delta^{2} \leq 1\right)$. The same assumption holds for $\hat{g}_{k i}(b, t)$ and $\tilde{g}_{k i}(b, t) .\left\{\hat{f}_{i k}(b, t)\right\}$ are available at $\mathcal{S}$, and $\left\{\hat{g}_{k i}(b, t)\right\}$ are available at the relays at time slot $(b, t)$. We introduce a parameter $\alpha \geq 0$ as the power exponent of the estimation error:

$$
\alpha \triangleq-\frac{\log \left(\delta^{2}\right)}{\log P} .
$$

Assume $\alpha$ has a well-defined limit when $P \rightarrow \infty$ so that it can be seen as the quality of instantaneous CSIT in the high SNR regime. From the perspective of DOF, $\alpha=0$ corresponds to the case with no instantaneous CSIT, while $\alpha \rightarrow \infty$ corresponds to the case with accurate instantaneous CSIT (i.e. perfect CSIT). Therefore, increasing $\alpha$ increases the quality of CSIT. Furthermore, since $\alpha>1$ implies that estimation error is negligible compared to noise, the instantaneous CSIT can be regarded as accurate from the DOF perspective [3]. Hence we only consider $\alpha \in[0,1]$ throughout the paper.
2) Accurate delayed CSIT: The accurate channel information can be fed back to the transmitter side after one block delay. Thus, at block $b$, the source knows the delayed CSI of the first hop $f_{i k}(b-1, t)$ and the relays know the delayed CSI of the second hop $g_{k i}(b-1, t), \forall t \in\{1,2, \ldots, T\}, k \in\{1,2\}$ and $i \in\{1,2, \ldots, I\}$.

\section{MAIN RESULTS}

We present our main results in this section.

Theorem 1: With mixed CSIT and $I \geq 3$ relays, the considered two-hop $2 \times I \times 2$ MISO broadcast network has sum optimal DOF $\frac{4+2 \alpha}{3}$, for $0 \leq \alpha \leq 1$.

Proof: The proof is presented in Section IV.

Remark 1: From Theorem 1, the considered two-hop network has the same sum DOF as the single-hop two-user BC. As we mentioned earlier, when $\alpha=0$ each transmitter has no information regarding the instantaneous channel condition. Only delayed CSIT is available. Following Theorem 1, the sum DOF is $\frac{4}{3}$. This result coincides with that shown in our earlier work [27]. With a positive $\alpha$, the sum DOF of the network is actually a linear function of $\alpha$ and is strictly larger than $\frac{4}{3}$. In addition, we can prove (see Proposition 1 in Appendix C) that, with only inaccurate instantaneous CSIT, the LBF-based amplify-forward relaying can achieve the DOF $2 \alpha$. Theorem 1 shows that having both forms of inaccurate CSIT provides extra DOF gain over having only one of them. When $\alpha=1$, the quality of the instantaneous CSIT is sufficiently good such that the sum DOF of the network is 2, same as that when perfect CSIT is available.

\section{Proof of Theorem 1: The SUM DOF of $2 \times I \times 2$ NETWORKS FOR $I \geq 3$}

In what follows, we present the proof of Theorem 1. To this end, we first find an upper bound on the sum DOF. Then an achievable scheme is proposed to show that the upper bound is tight.

\section{A. Upper Bound}

To prove the converse of Theorem 1, we assume that a genie provides all source messages to the relays non-causally. This assumption would not degrade the capacity region of the considered network. Now the new network's sum DOF is bounded by only the second hop. Assuming full cooperation at relays, the second hop works as a single-hop MISO BC with an $I$-antenna source and 2 single-antenna receivers. Following [11] [12], when $I \geq 2$, the sum DOF of this $I \times 2$ MISO BC with mixed CSIT is $\frac{4+2 \alpha}{3}$. Thus, $\frac{4+2 \alpha}{3}$ also serves as the sum DOF upper bound for the considered network.

It is worth noting that, based on the same arguement, we can show that the DOF region of the considered network can be outer bounded as follows, directly following the outer bound of the single-hop MISO BC [11] [12]. Let $\left(d_{1}, d_{2}\right)$ represent the DOF for messages intended for $\mathcal{D}_{1}$ and $\mathcal{D}_{2}$, respectively. Then, we have

$$
\begin{aligned}
d_{1}, d_{2} & \leq 1 \\
d_{1}+2 d_{2} & \leq 2+\alpha, \\
2 d_{1}+d_{2} & \leq 2+\alpha .
\end{aligned}
$$


The polygon given by the above outer bounds can be shown to be optimal by achieving all the vertices of the polygon: $(1,0),(0,1),(\alpha, 1),(1, \alpha)$ and $\left(\frac{2+\alpha}{3}, \frac{2+\alpha}{3}\right)$. In this proof, we focus on achieving the point $d_{1}=d_{2}=\frac{2+\alpha}{3}$, which yields the maximum sum DOF and implies the achievability of the other vertices of the region.

To facilitate presentation, we divide the process of designing the achievable scheme into two steps. We first propose an RIA method to make use of only the delayed CSIT. We show that, without taking instantaneous CSIT into consideration, sum DOF $\frac{4}{3}$ is achievable in the $2 \times I \times 2$ MISO broadcast network. Afterwards, a new LBF-based protocal is applied on top of the RIA scheme to take advantage of the available instantaneous CSIT so that the sum DOF upper bound $\frac{4+2 \alpha}{3}$ can be achieved.

\section{B. Retrospective Interference Alignment with Delayed CSIT}

In this subsection, we present a transmission scheme, modified from our previous work [27], that aims to make use of only delayed CSIT. The modification is critical for the later integration with instantaneous CSIT. From the previous subsection, it is seen that in this case the maximal achievable DOF is $\frac{4}{3}$. We show that activating only two relays $\left(\mathcal{R}_{1}\right.$ and $\mathcal{R}_{2}$ ) suffices to attain this result. Without loss of generality, we assume each Gaussian codeword is transmitted within one block length, and we drop the time slot index $t$ in the indices pair $(b, t)$ hereafter to simplify the notations, e.g., we simplify $X(b, t)$ into $X(b), F_{i}(b, t)$ into $F_{i}(b)$, etc.

Consider a multi-round transmission scheme. Let integer $L \geq 1$ represent the round number. We will show that $4 L$ independent messages from the source can be delivered in $3 L+3$ blocks. Then when $L \rightarrow \infty$, the sum DOF $\frac{4 L}{3 L+3} \rightarrow \frac{4}{3}$ can be achieved asymptotically. We start introducing the transmission scheme from the first round, which takes the blocks $1,2,3$ at the first hop and the following three blocks $(4,5,6)$ at the second hop. Let $X_{o}^{[k]}=\left[\begin{array}{ll}x_{o, 1}^{[k]} & x_{o, 2}^{[k]}\end{array}\right]^{T}$ represent the codeword intended for $\mathcal{D}_{k}(k \in\{1,2\})$, where $x_{o, 1}^{[k]}$ and $x_{o, 2}^{[k]}$ are two independent Gaussian codewords representing messages $M_{k 1}$ and $M_{k 2}$, respectively. We show $X_{o}^{[k]}$ can be delivered to $\mathcal{D}_{k}$ with $2 \mathrm{DOF}$ after the first round. Assume equal transmission power $P$ at each terminal, which is omitted for simplicity.

1) Transmission at the first hop: The transmission signal of the first block can be written as $X(1)=X_{o}^{[1]}+X_{o}^{[2]}$. Use $F_{k}^{T}$ to denote the channel vector from $\mathcal{S}$ to $\mathcal{R}_{k}$ during the first block (i.e. $F_{k}^{T}(1)$ ). The received signals at relays are displayed in Table I, excluding noise for concise. After block $1, F_{2}^{T} X_{o}^{[1]}$ and $F_{1}^{T} X_{o}^{[2]}$ are desired by both relays for decoding, thus are termed as common-desired signals. Because of the delayed CSIT, at the beginning of block 2 the source knows the values of $F_{1}^{T}$ and $F_{2}^{T}$. It constructs its transmission signals for the following two blocks as

$$
X(2)=\left[\begin{array}{ll}
F_{2}^{T} X_{o}^{[1]} & 0
\end{array}\right]^{T}, \quad X(3)=\left[\begin{array}{ll}
0 & F_{1}^{T} X_{o}^{[2]}
\end{array}\right]^{T} .
$$

The received signals of the relays (excluding noise) are displayed in Table I. Using $y_{R_{1}}(1)-\frac{y_{R_{1}}(3)}{f_{12}(3)}$ and $y_{R_{1}}(2)$, relay $\mathcal{R}_{1}$ obtains sufficient independent equations to decode the two messages in $X_{o}^{[1]}$. It also knows an interference term $F_{1}^{T} X_{o}^{[2]}$, which is a linear combination of the two codewords in $X_{o}^{[2]}$. Similarly, relay $\mathcal{R}_{2}$ obtains sufficient independent equations to decode the two codewords in $X_{o}^{[2]}$ using $y_{R_{2}}(1)-\frac{y_{R_{2}}(2)}{f_{21}(2)}$ and $y_{R_{2}}(3)$. A linear combination of the two codewords in $X_{o}^{[1]}$ is also available at $\mathcal{R}_{2}$ as an interference term $F_{2}^{T} X_{o}^{[1]}$. The interference terms at the relays will play an important role in the transmission of the second hop.

2) Transmission at the second hop: During the fourth block, the relays respectively form their transmission signals $x_{R_{1}}(4)$ and $x_{R_{2}}(4)$ using the decoded codewords and the interference terms, as shown in Table II (excluding residue noise for brevity). Define $A_{1}^{T}=g_{11}(4) F_{1}^{T}+g_{12}(4) F_{2}^{T}$ and $A_{2}^{T}=g_{21}(4) F_{1}^{T}+g_{22}(4) F_{2}^{T}$. Clearly, $A_{1}$ and $A_{2}$ are linearly independent, almost surely. Again, $A_{2}^{T} X_{o}^{[1]}$ and $A_{1}^{T} X_{o}^{[2]}$ are desired by both destinations for decoding. Because of the delayed CSIT, at the beginning of block 5, $A_{1}$ and $A_{2}$ are known at the relays. Then during the fifth and the sixth blocks, $x_{R_{k}}(5)$ and $x_{R_{k}}(6)(k=1,2)$, the transmission signals of the relays are computed as shown in Table II. Clearly, using $y_{1}(4)-\frac{y_{1}(6)}{g_{12}(6)}$ and $y_{1}(5), \mathcal{D}_{1}$ obtains sufficient linearly independent equations to recover the messages in $X_{o}^{[1]} \cdot \mathcal{D}_{2}$ also has sufficient linearly independent equations to recover the messages in $X_{o}^{[2]}$, using $y_{2}(4)-\frac{y_{2}(5)}{g_{21}(5)}$ and $y_{2}(6)$. The first round of transmission manages to deliver 4 messages in 6 blocks. By induction, the $l$ th $(l \in\{1,2, \ldots, L\})$ round transmission takes the blocks $3 l-2$ to $3 l$ at the first hop and the blocks $3 l+1$ to $3 l+3$ at the second hop to deliver 4 messages. Therefore, in total $3 l+3$ blocks are used to transmit $4 l$ codewords. The sum DOF $\frac{4}{3}$ can be achieved asymptotically. We can can attain the following result.

Lemma 1: With delayed CSIT and $I \geq 2$ relays, the sum DOF $\frac{4}{3}$ can be achieved for the considered 2-hop $2 \times I \times 2$ network.

In the next subsection, we introduce a new LBF-based scheme on top of the above transmission to take advantage of the available instantaneous CSIT and obtain higher DOF. Before proceeding, it is worth noting that the transmission process of the above RIA scheme can be summarized as two phases. In phase one, transmission signals are blindly broadcasted from transmitters and thus are superimposed at each receiver. Both receivers require more proper linear combinations of these codewords (i.e. the common-desired signals) to decode their expected messages. The critical point is that the common-desired signals can be recovered at transmitters with the help of delayed CSIT. Thus in the second phase, they are delivered to the receivers to facilitate decoding. This perspective of viewing the transmission is crucial to the integration of instantaneous CSIT.

\section{Integrating Instantaneous CSIT - An Overview}

By integrating instantaneous CSIT, we aim to show that in the considered $2 \times I \times 2(I \geq 3)$ MISO broadcast network, the sum DOF $\frac{4+2 \alpha}{3}$ can be achieved. In this subsection, we provide an overview of the transmission scheme and address the key problems in the scheme design. To help understand 
TABLE I

RIA AT THE FIRST HOP [4]

\begin{tabular}{c|c|c|c}
\hline$b$ & 1 & 2 & 3 \\
\hline$X(b)$ & $X_{o}^{[1]}+X_{o}^{[2]}$ & {$\left[F_{2}^{T} X_{o}^{[1]} 0\right]^{T}$} & {$\left[0 F_{1}^{T} X_{o}^{[2]}\right]^{T}$} \\
\hline$y_{R_{1}}(b)$ & $F_{1}^{T}\left(X_{o}^{[1]}+X_{o}^{[2]}\right)$ & $f_{11}(2) F_{2}^{T} X_{o}^{[1]}$ & $f_{12}(3) F_{1}^{T} X_{o}^{[2]}$ \\
\hline$y_{R_{2}}(b)$ & $F_{2}^{T}\left(X_{o}^{[1]}+X_{o}^{[2]}\right)$ & $f_{21}(2) F_{2}^{T} X_{o}^{[1]}$ & $f_{22}(3) F_{1}^{T} X_{o}^{[2]}$ \\
\hline
\end{tabular}

TABLE II

RIA AT THE SECOND HOP

\begin{tabular}{c|c|c|c}
\hline$b$ & 4 & 5 & 6 \\
\hline$x_{R_{1}}(b)$ & $F_{1}^{T}\left(X_{o}^{[1]}+X_{o}^{[2]}\right)$ & $A_{2}^{T} X_{o}^{[1]}$ & 0 \\
\hline$x_{R_{2}}(b)$ & $F_{2}^{T}\left(X_{o}^{[1]}+X_{o}^{[2]}\right)$ & 0 & $A_{1}^{T} X_{o}^{[2]}$ \\
\hline$y_{1}(b)$ & $A_{1}^{T}\left(X_{o}^{[1]}+X_{o}^{[2]}\right)$ & $g_{11}(5) A_{2}^{T} X_{o}^{[1]}$ & $g_{12}(6) A_{1}^{T} X_{o}^{[2]}$ \\
\hline$y_{2}(b)$ & $A_{2}^{T}\left(X_{o}^{[1]}+X_{o}^{[2]}\right)$ & $g_{21}(5) A_{2}^{T} X_{o}^{[1]}$ & $g_{22}(6) A_{1}^{T} X_{o}^{[2]}$ \\
\hline
\end{tabular}

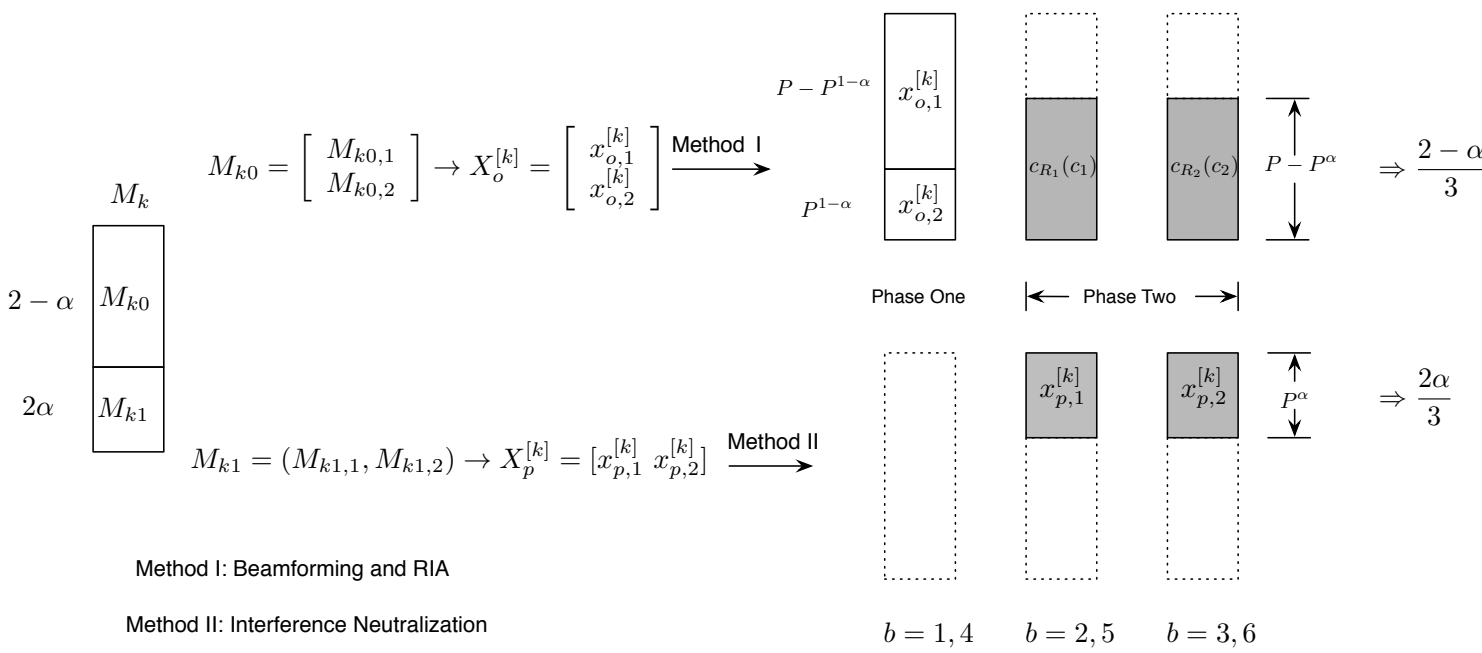

Fig. 2. Overview of the achievable scheme.

the structure of our proposed transmission approach, we start from the intuition behind the achieved DOF result, which can be represented as follows:

$$
d=\frac{4+2 \alpha}{3}=\underbrace{\frac{2 \times(2-\alpha)}{3}}_{\text {Method I }}+\underbrace{\frac{2 \times 2 \alpha}{3}}_{\text {Method II }} .
$$

This implies that we shall split the message into two parts, each of which is transmitted by a specific method. By keeping the similar 3-block transmission structure as introduced in the previous subsection, method I, which occupies two phases, aims to beamform the transmission signals based on the instantaneous CSIT in the first phase such that the power of common-desired signals (interference) is reduced. Consequently, in the second phase, a reduced power level is sufficient for delivering the common-desired signals. Essentially, method $I$ captures the idea of combining beamforming and RIA. The purpose of method II is to convey some extra information by the residue power in phase two, based on the instantaneous CSIT. Specifically, only amplify-forward relaying is carried out in the method II, which exploits the idea of interference neutralization [19].

In what follows, we raise two key questions in the scheme design. The proposed solution to the problems addressed in the questions constitutes the core of our achievable scheme:

Question 1: In method I, how can we reduce the interference power based on the instantaneous CSIT, especially at the second hop by distributed relays?

In [10] [12], the authors proposed a method based on the LBF and asymmetric power allocation for the single-hop BC. That can be applied to reduce the interference power at the first hop. For the second hop, we propose a new LBF-based scheme at distributed relays which shows that the same purpose can be achieved without cooperation at relays. The detailed approach will be presented in the phase one of the second hop.

Question 2: In method II, how can we deliver extra messages with the residue power in the two-hop network?

In the second phase, we superpose the extra messages on the common-desired signals with the residue power. After the common-desired signals are decoded at each relay, the extra messages are amplified and sent to destinations. The amplification factors are designed based on the idea of interference neutralization, which can be interpreted in the following toy example. Consider there exist 3 relays in the network, and two messages at the transmitter, each transmitted by one antenna and intended for one receiver. Let $a_{1}, a_{2}$ and $a_{3}$ denote the 
power amplification factors. In order to neutralize interference (i.e., eliminate the interference on the air) at $\mathcal{D}_{1}$ and $\mathcal{D}_{2}$, one should have

$$
\begin{aligned}
& g_{11} a_{1} f_{12}+g_{12} a_{2} f_{22}+g_{13} a_{3} f_{32}=0, \\
& g_{21} a_{1} f_{11}+g_{22} a_{2} f_{21}+g_{23} a_{3} f_{31}=0 .
\end{aligned}
$$

The above equation group has nonzero solutions for $\left[\begin{array}{lll}a_{1} & a_{2} & a_{3}\end{array}\right]$ almost surely, provided the channel coefficients are chosen from some continuous distribution. As we will show in the second phase of transmission, based on the instantaneous CSIT $\tilde{g}_{i j}$, this amplify-forward method can still introduce a DOF $\frac{4 \alpha}{3}$, though the interferences are not perfectly neutralized.

As discussed above, the operations at relays are critical to the achievablity. By keeping the 3-block multi-round transmission structure similar to that shown in Table I and II, we provide an outline for the proof.

- Rate splitting: Let $M_{k}=\left(M_{k 0}, M_{k 1}\right) \in\left[1: 2^{T R_{k}}\right](k=$ $1,2)$ represent the message intending for $\mathcal{D}_{k}$, where the independent messages $M_{k 0} \in\left[1: 2^{T R_{o}^{[k]}}\right]$ and $M_{k 1} \in$ $\left[1: 2^{T R_{p}^{[k]}}\right]$ with $R_{k}=R_{o}^{[k]}+R_{p}^{[k]}$. Intuitively, $M_{k 0}$ represents the message to be transmitted by mathod I, and $M_{k 1}$ represents the extra information to be delivered by method II.

- Encoding: For each message $M_{k 0}$, we split it into two sub-messages $M_{k 0,1}$ and $M_{k 0,2} . M_{k 0, i}$ is encoded into a Gaussian codeword $x_{o, i}^{[k]}(i \in\{1,2\})$. Let $X_{o}^{[k]}=$ $\left[\begin{array}{ll}x_{o, 1}^{[k]} & x_{o, 2}^{[k]}\end{array}\right]^{T}$, which can be transmitted by 2 antennas in one block. We split each message $M_{k 1}$ into two submessages $M_{k 1,1}$ and $M_{k 1,2}$ with rates $R_{p 1}^{[k]}$ and $R_{p 2}^{[k]}$, respectively. Thus, $R_{p}^{[k]}=R_{p 1}^{[k]}+R_{p 2}^{[k]} . M_{k 1, i}$ is encoded into a Gaussian codeword $x_{p, i}^{[k]}$. Let $X_{p}^{[k]}=\left[x_{p, 1}^{[k]} x_{p, 2}^{[k]}\right]$. The transmission of $X_{p}^{[k]}$ covers two blocks.

- Consider the first round of transmission, which takes the blocks 1 to 3 at the first hop and the blocks 4 to 6 at the second hop. We will show that $X_{o}^{[k]}$ can be delivered to $\mathcal{D}_{k}$ with the rate $R_{o}^{[k]} \geq(2-\alpha) \log (P)+o(\log (P))$. In addition, $X_{p}^{[k]}$ can be delivered to $\mathcal{D}_{k}$ with the rate $R_{p}^{[k]} \geq 2 \alpha \log (P)+o(\log (P))$. Therefore, we have $R_{k}=$ $R_{o}^{[k]}+R_{p}^{[k]} \geq(2+\alpha) \log (P)+o(\log (P))$.

- The whole transmission works in a pipeline fashion with multiple rounds, as we interpreted in the delayed CSIT case. Asymptotically, each round of transmission takes 3 blocks time. Therefore, the achieved sum DOF is $d=$ $\lim _{P \rightarrow \infty} \frac{R_{1}+R_{2}}{3 \log (P)}=\frac{4+2 \alpha}{3}$.

The overview of the achievable scheme is illustrated in Fig. 2, with the interpretation of message splitting and the corresponding transmission approach. The transmission details of two hops are presented in the following two subsections, respectively. Note that we omit the power scaling factors as long as they do not affect the DOF [4].

\section{The First Hop - A MISO BC Approach}

We treat the first hop as a MISO BC and adopt a similar transmission approach proposed in [10] [12]. The goal of the first hop is to let relays $\mathcal{R}_{1}$ and $\mathcal{R}_{2}$ decode $X_{o}^{[1]}$ and
$X_{o}^{[2]}$, respectively. Meanwhile, relay $\mathcal{R}_{3}$ only listens to the transmission without decoding either $X_{O}^{[1]}$ or $X_{O}^{[2]}$.

Phase One - Transmit $X_{o}^{[k]}$ : The first phase takes the block 1. Let $\mathbf{P}$ represent the $2 \times 2$ diagonal power allocation matrix. $\mathbf{V}_{k}=\left[\begin{array}{ll}V_{k, 1} & V_{k, 2}\end{array}\right]$ represents the $2 \times 2$ unitary beamforming matrix for $X_{o}^{[k]}(k \in\{1,2\})$, where $V_{k, 1}$ and $V_{k, 2}$ are two orthonormal column vectors. Then, the transmitted signal at the source $\mathcal{S}$ is

$$
X(1)=\mathbf{V}_{1} \mathbf{P}^{\frac{1}{2}} X_{o}^{[1]}+\mathbf{V}_{2} \mathbf{P}^{\frac{1}{2}} X_{o}^{[2]} .
$$

We set the power allocation matrix to be $\mathbf{P}=$ $\operatorname{diag}\left\{P-P^{1-\alpha}, P^{1-\alpha}\right\}$ similar to [10]. The received signals at relays can be represented as

$$
\begin{aligned}
& y_{R_{1}}(1)=F_{1}^{T}(1) \mathbf{V}_{1} \mathbf{P}^{\frac{1}{2}} X_{o}^{[1]}+\sigma_{R_{1}}+z_{1}(1), \\
& y_{R_{2}}(1)=F_{2}^{T}(1) \mathbf{V}_{2} \mathbf{P}^{\frac{1}{2}} X_{o}^{[2]}+\sigma_{R_{2}}+z_{2}(1), \\
& y_{R_{3}}(1)=L_{1} X_{o}^{[1]}+L_{2} X_{o}^{[2]}+z_{3}(1),
\end{aligned}
$$

where $\sigma_{R_{1}}=F_{1}^{T}(1) \mathbf{V}_{2} \mathbf{P}^{\frac{1}{2}} X_{o}^{[2]}, \sigma_{R_{2}}=F_{2}^{T}(1) \mathbf{V}_{1} \mathbf{P}^{\frac{1}{2}} X_{o}^{[1]}$, $L_{1}=F_{3}^{T}(1) \mathbf{V}_{1} \mathbf{P}^{\frac{1}{2}}$ and $L_{2}=F_{3}^{T}(1) \mathbf{V}_{2} \mathbf{P}^{\frac{1}{2}} \cdot \sigma_{R_{k}}$ can be seen as the interference term at $\mathcal{R}_{k}$. The beamforming design at $\mathcal{S}$ aims for reducing the power of $\sigma_{R_{k}}$ based on the instantaneous CSIT $\hat{F}_{k}(t)$. Specifically, the beamforming matrices are chosen such that $V_{1,1} \perp \hat{F}_{2}(1)$ and $V_{2,1} \perp \hat{F}_{1}(1)$. By this means, the signal sent with the power $P-P^{1-\alpha}$ is partially zero-forced at the unintended user. Because each Gaussian codeword experiences sufficiently many channel coefficients in one block, the average power of interference can be shown to be $O\left(P^{1-\alpha}\right)$ [10] [11]:

$$
\mathbb{E}\left(\left|\sigma_{R_{k}}\right|^{2}\right)=O\left(P^{1-\alpha}\right) .
$$

Quantize the interference $\sigma_{R_{k}}$ : After phase one, because of the delayed CSI feedback, $F_{k}(1)$ can be obtained perfectly at the source. Thus the interference $\sigma_{R_{k}}$ can be recovered by the source. In order to decode $X_{o}^{[1]}$ and $X_{o}^{[2]}$ at relays, $\sigma_{R_{1}}$ and $\sigma_{R_{2}}$ are desired by both $\mathcal{R}_{1}$ and $\mathcal{R}_{2}$. We aim to deliver them to relays in phase two as common messages. Since the average power of $\sigma_{R_{k}}$ is $O\left(P^{1-\alpha}\right)$, we transmit them in a digital fashion for efficiency. We quantize $\sigma_{R_{k}}$ into $\hat{\Sigma}_{R_{k}}$. The index of $\hat{\Sigma}_{R_{k}}$ can be represented in $R_{k}^{\prime}$ bits, which is encoded into the Gaussian codeword $c_{R_{k}}$. As shown in [11] and [12], by setting the distortion level to 1 , which does not effect the DOF, the number of quantization bits can be upper bounded as $R_{k}^{\prime} \leq(1-\alpha) \log (P)+o(\log (P))$.

Phase Two - Transmit $c_{R_{k}}$ and $X_{p}^{[k]}$ : This phase takes the blocks 2 and 3 . The purpose of this phase is to deliver $c_{R_{k}}$ ( $k \in\{1,2\}$ ) to all relays as the common message. Meanwhile, we send new codewords $X_{p}^{[k]}=\left[x_{p, 1}^{[k]} x_{p, 2}^{[k]}\right]$ for destinations $\mathcal{D}_{k}$, for $k \in\{1,2\}$. Specifically, the transmission signals are designed based on superposition coding technique. During the blocks $b=2,3$, the transmission signals at $\mathcal{S}$ are

$$
X(b)=\sqrt{P-P^{\alpha}}\left[\begin{array}{l}
c_{R_{b-1}} \\
c_{R_{b-1}}
\end{array}\right]+\sqrt{P^{\alpha}}\left[\begin{array}{c}
x_{p, b-1}^{[1]} \\
x_{p, b-1}^{[2]}
\end{array}\right] .
$$

For instance, during the block $2, c_{R_{1}}$ is transmitted from both antennas with power $P-P^{\alpha}$, whereas two distinct 

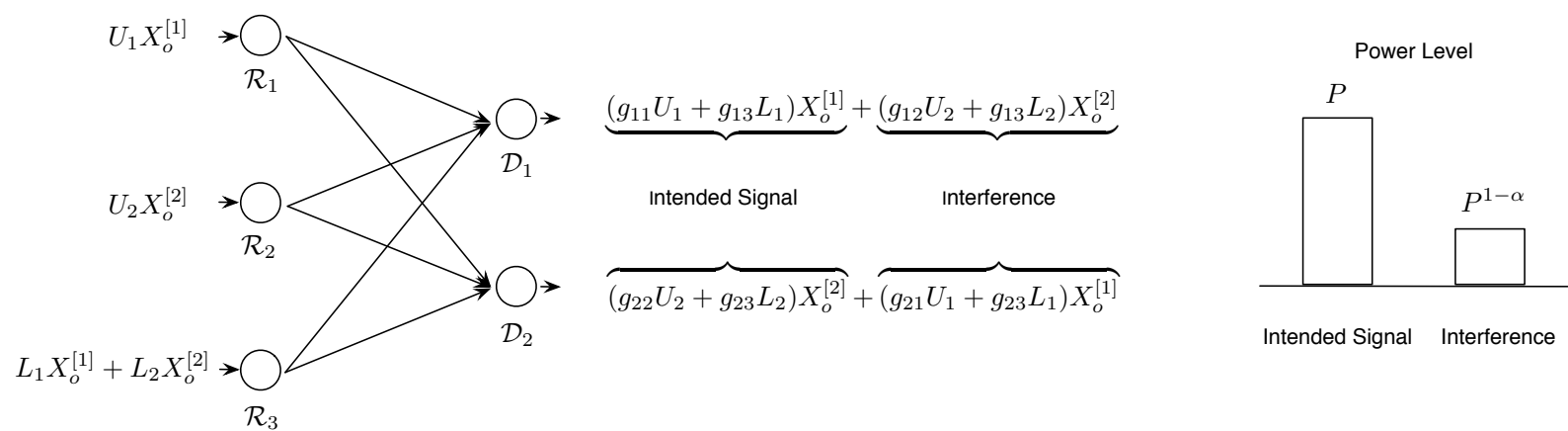

Intended Signal Interference

Fig. 3. The main idea of phase one at the second hop.

new codewords $x_{p, 1}^{[k]}$ are transmitted from the source with the residue power. Then, the received signals at relays are

$$
\begin{aligned}
y_{R_{i}}(b)= & F_{i}^{T}(b)\left(\sqrt{P-P^{\alpha}}\left[\begin{array}{l}
c_{R_{b-1}} \\
c_{R_{b-1}}
\end{array}\right]+\sqrt{P^{\alpha}}\left[\begin{array}{l}
x_{p, b-1}^{[1]} \\
x_{p, b-1}^{[2]}
\end{array}\right]\right) \\
& +z_{i}(b), i=1,2,3 .
\end{aligned}
$$

Decoding at relays: The decoding process at relays works as follows.

- Decode $c_{R_{k}}$ : All relays first try to decode $c_{R_{1}}$ and $c_{R_{2}}$ from the received signals in phase two (14). Treating $X_{p}^{[k]}$ as noise, and because the signal $\left(c_{R_{k}}\right)$ to interference-plus-noise ratio (SINR) is on the level of $O\left(\frac{P-P^{\alpha}}{P^{\alpha}}\right)=O\left(P^{1-\alpha}\right)$, the following rate can be achieved for decoding $c_{R_{k}}$ :

$$
R_{c}^{\prime}=(1-\alpha) \log (P)+o(\log (P)) .
$$

- Recover $\hat{\Sigma}_{R_{k}}$ from $c_{R_{k}}$ : It is possible to recover the index of $\hat{\Sigma}_{R_{k}}$ if $R_{k}^{\prime} \leq R_{c}^{\prime}$. Thus, $R_{c}^{\prime}$ and $R_{k}^{\prime}$ can be chosen properly to satisfy this constraint.

- Decode $X_{o}^{[k]}$ at relay $\mathcal{R}_{k}$ : Relay $\mathcal{R}_{1}$ and $\mathcal{R}_{2}$ try to decode $X_{o}^{[1]}$ and $X_{o}^{[2]}$, respectively. With $\hat{\Sigma}_{R_{k}}$, combining the received signal in the first phase, $\mathcal{R}_{k}$ can decode $X_{o}^{[k]}$ in the following rate (see details in [11] [12]):

$$
R_{o}^{[k]} \geq(2-\alpha) \log (P)+o(\log (P)) .
$$

- $\mathcal{R}_{i}(i \in\{1,2,3\})$ subtracts the decoded messages $c_{R_{k}}$ from the received signal (14). The remaining signals will be transmitted later at the second hop.

\section{E. The Second Hop - Settling Questions 1 and 2}

The transmission design of the second hop aims to answer the two key questions. During phase one $(b=4)$, we propose a new LBF-based scheme at distributed relays to reduce the power of $X_{o}^{[k]}$ at $\mathcal{D}_{j}$ to the level of $O\left(P^{1-\alpha}\right)(k \neq j)$. During phase two $(b=5,6)$, an amplify-forward relaying scheme is applied for neutralizing the interference caused by the messages $X_{p}^{[k]}$ at $\mathcal{D}_{j}$. We summarize the useful signals at relays after the transmission at the first hop, with the noise term being omitted for simplicity. From phase one,

$$
\begin{aligned}
& \mathcal{R}_{1}: X_{o}^{[1]}, \\
& \mathcal{R}_{2}: X_{o}^{[2]}, \\
& \mathcal{R}_{3}: L_{1} X_{o}^{[1]}+L_{2} X_{o}^{[2]} .
\end{aligned}
$$
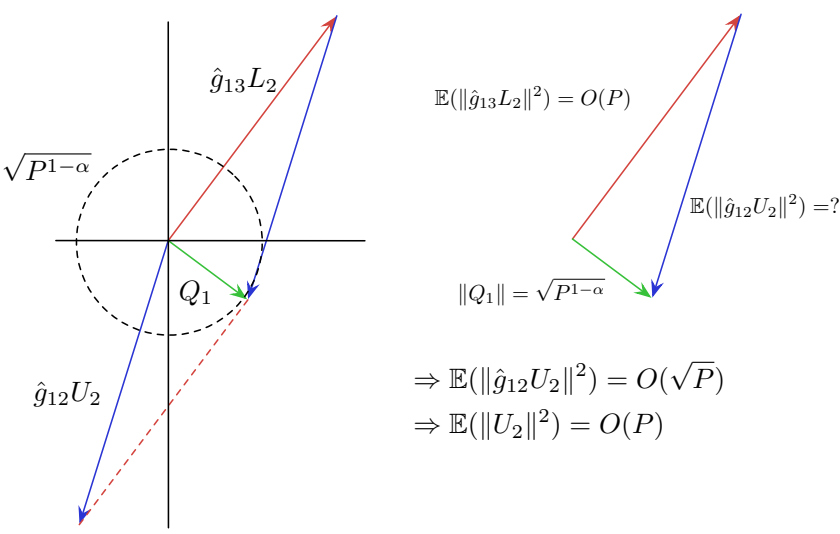

Fig. 4. The illustration of interference alignment at destination $\mathcal{D}_{1}$.

And from phase two, $b=2,3$

$$
\mathcal{R}_{i}: \sqrt{P^{\alpha}} F_{1}^{T}(b)\left[\begin{array}{c}
x_{p, b-1}^{[1]} \\
x_{p, b-1}^{[2]}
\end{array}\right], i \in\{1,2,3\}
$$

(17) and (18) will be used in the phase one and two, respectively, by two different methods. The details are presented as follows.

Phase One - Transmit $X_{o}^{[k]}$ : The key idea of this phase is to design two $1 \times 2$ beamforming vectors $U_{1}$ and $U_{2}$ at relay $\mathcal{R}_{1}$ and $\mathcal{R}_{2}$, respectively, for transmitting the signals (17). The solution of $U_{1}$ and $U_{2}$ essentially solves the problem that we addressed in Question 1: to reduce the interference power caused by $X_{o}^{[k]}$ at $\mathcal{D}_{j}$ to the power level $O\left(P^{1-\alpha}\right)$. We illustrate our main purpose in Fig. 3. The technical details are presented as follows.

The transmission signals at relays are

$$
\begin{aligned}
& x_{R_{1}}(4)=U_{1} X_{o}^{[1]}, \quad x_{R_{2}}(4)=U_{2} X_{o}^{[2]}, \\
& x_{R_{3}}(4)=y_{R_{3}}(1)=L_{1} X_{o}^{[1]}+L_{2} X_{o}^{[2]}+z_{3}(1),
\end{aligned}
$$

In particular, $\mathcal{R}_{3}$ simply transmits its received signal during block 1 . Note that the transmission power is implicitly included in the beamforming vectors here. Omitting the block 
indices, the received signals at destinations can be written as:

$$
\begin{aligned}
& y_{1}=\underbrace{\left(g_{11} U_{1}+g_{13} L_{1}\right)}_{H_{11}} X_{o}^{[1]}+\underbrace{\left(g_{12} U_{2}+g_{13} L_{2}\right)}_{H_{12}} X_{o}^{[2]}+n_{1}^{\prime}, \\
& y_{2}=\underbrace{\left(g_{21} U_{1}+g_{23} L_{1}\right)}_{H_{21}} X_{o}^{[1]}+\underbrace{\left(g_{22} U_{2}+g_{23} L_{2}\right)}_{H_{22}} X_{o}^{[2]}+n_{2}^{\prime},
\end{aligned}
$$

where $n_{k}^{\prime}=n_{k}+g_{k 3} z_{3}(k=1,2)$ is the effective noise. Let us define the interference terms as $\sigma_{1}=H_{12} X_{o}^{[2]}$ and $\sigma_{2}=H_{21} X_{o}^{[1]}$. For designing the beamforming vectors $U_{k}$, we essentially work on the following problem.

Find $U_{k}, k \in\{1,2\}$

s.t. $\quad \mathrm{C} 1: \mathbb{E}\left(\log \left(\left|\sigma_{k}\right|^{2}\right)\right)=(1-\alpha) \log (P)+o(\log (P))$,

C2: $\mathbb{E}\left(\left\|H_{k k}\right\|^{2}\right)=O(P)$,

C3: $H_{1 k}$ is independent to $H_{2 k}$,

C4: $\mathbb{E}\left(\left\|U_{k}\right\|^{2}\right)=O(P)$

Constraints $\mathrm{C} 1$ and $\mathrm{C} 2$ are important because they capture the idea of reducing the power of interference to $O\left(P^{1-\alpha}\right)$, whereas keeping the power of intended signals as $O(P)$. C3 implies that the interference terms should provide new information (linear combination) for decoding $X_{o}^{[k]}$ in the second phase, delivered as common-desired signals. $\mathrm{C} 4$ is the power constraint on beamforming vectors. In the following, we present the mathematical details for the feasible $U_{k}$ to satisfy all the conditions.

Let $Q_{k}$ represent the $1 \times 2$ reference vector, $k \in\{1,2\}$. By introducing the reference vectors, we aim to align the interference along the direction of $Q_{k}$ at destination $\mathcal{D}_{k}$ based on the inaccurate instantaneous CSIT, with the power level $P^{1-\alpha}$. One should have:

$$
\begin{aligned}
& \hat{g}_{12} U_{2}+\hat{g}_{13} L_{2}=Q_{1}, \\
& \hat{g}_{21} U_{1}+\hat{g}_{23} L_{1}=Q_{2}, \\
& \left\|Q_{k}\right\|^{2}=P^{1-\alpha}, \quad k=1,2,
\end{aligned}
$$

Therefore, $Q_{k}$ can be chosen as any arbitrary radius of the circle shown in Fig. 4. Furthermore, we impose that $Q_{1} \perp L_{2}$ and $Q_{2} \perp L_{1}$. As we show in the sequel, this enforcement offers a feasible solution of $U_{k}$.

As illustrated in Fig. 4, at $\mathcal{D}_{1}$, the interferences coming from $\mathcal{R}_{2}$ and $\mathcal{R}_{3}$ through two independent paths, namely, $U_{2} X_{o}^{[2]}$ and $L_{2} X_{o}^{[2]}$ are aligned along the direction $Q_{1}$ with the power $P^{1-\alpha}$. We can also interpret the idea as finding the hypotenuse $\left(\hat{g}_{12} U_{2}\right)$ in the right triangle with two sides $\left(\hat{g}_{13} L_{2}\right.$ and $Q_{1}$ ) in the two dimensional Euclidean space. Therefore, the beamforming vectors $U_{1}$ and $U_{2}$ can be chosen by solving the equations (21) and (22):

$$
\begin{aligned}
& U_{1}=\hat{g}_{21}^{-1}\left(Q_{2}-\hat{g}_{23} L_{1}\right), \\
& U_{2}=\hat{g}_{12}^{-1}\left(Q_{1}-\hat{g}_{13} L_{2}\right) .
\end{aligned}
$$

It can be shown that the average power of $U_{k} X_{o}^{[k]}$ lies on the level of $P$, thus ignoring the power scaling factors does not affect the DOF analysis. C4 is satisfied. Briefly speaking, we manage to use the beamforming vectors with power level $P$ such as $U_{k}$ and $L_{k}$, to align the interference into power level $P^{1-\alpha}$. Fig. 4 provides the intuition of the beamforming designs. Constraints $\mathrm{C} 1, \mathrm{C} 2$ and $\mathrm{C} 3$ are checked in the following.

Substituting (23-24) into (19-20), we have

$$
\begin{aligned}
& H_{11}=\frac{g_{11}}{\hat{g}_{21}} Q_{2}+\left(g_{13}-\frac{g_{11} \hat{g}_{23}}{\hat{g}_{21}}\right) L_{1}, \\
& H_{22}=\frac{g_{22}}{\hat{g}_{12}} Q_{1}+\left(g_{23}-\frac{g_{22} \hat{g}_{13}}{\hat{g}_{12}}\right) L_{2}, \\
& H_{12}=\frac{g_{12}}{\hat{g}_{12}} Q_{1}+\left(g_{13}-\frac{g_{12} \hat{g}_{13}}{\hat{g}_{12}}\right) L_{2}, \\
& H_{21}=\frac{g_{21}}{\hat{g}_{21}} Q_{2}+\left(g_{23}-\frac{g_{21} \hat{g}_{23}}{\hat{g}_{21}}\right) L_{1} .
\end{aligned}
$$

We observe that $H_{11}$ and $H_{21}$ are linearly independent almost surely. Thus if $\mathcal{D}_{1}$ can obtain $H_{21} X_{O}^{[1]}$ then combining $H_{11} X_{o}^{[1]}$, it is equivalent to a MIMO channel (see Appendix B) for decoding $X_{o}^{[1]}$. Similarly, if $\mathcal{D}_{2}$ can obtain $H_{12} X_{o}^{[2]}$, then combining with $H_{22} X_{O}^{[2]}$ it is also equivalent to a MIMO channel for decoding $X_{o}^{[2]}$. Therefore, C3 is satisfied. For C1, we calculate the second moment of interference with respect to logarithm (please refer to Appendix A)

$$
\mathbb{E}\left(\log \left(\left|\sigma_{k}\right|^{2}\right)\right) \leq(1-\alpha) \log (P)+o(\log (P)) .
$$

And meanwhile, it is readily shown that $\mathrm{C} 2$ is satisfied based on $H_{11}$ and $H_{22}$. Therefore, all the four constraints are so far satisfied by (23) and (24).

Quantizing the interference $\sigma_{k}$ : After the block 4, due to delayed CSIT, $H_{12}$ and $H_{21}$ can be obtained by all relays. In particular, because $X_{o}^{[1]}$ and $X_{o}^{[2]}$ can be decoded by $\mathcal{R}_{1}$ and $\mathcal{R}_{2}$, respectively, $\sigma_{1}$ and $\sigma_{2}$ can be recovered by $\mathcal{R}_{2}$ and $\mathcal{R}_{1}$, respectively. $\sigma_{k}$ will be transmitted in the second phase in a quantized form. Similar to the operations at the first hop, $\sigma_{k}$ is quantized into $\hat{\Sigma}_{k}$, and then the index of $\hat{\Sigma}_{k}$ containing $R_{\sigma_{k}}$ viewed as a message is encoded into a Gaussian codeword $c_{k}$. Due to (25), we have (by setting the distortion level to 1) [11]

$$
R_{\sigma_{k}} \leq(1-\alpha) \log (P)+o(\log (P)) .
$$

$c_{k}$ will be transmitted in the following phase for both destinations as common messages.

Phase Two - Transmit $c_{k}$ and $X_{p}^{[k]}$ : Phase two takes the blocks 5 and 6 . The goal is to deliver $c_{k}$ to both destinations for decoding $X_{o}^{[k]}$, and also to deliver $X_{p}^{[k]}$ to $\mathcal{D}_{k}$. Briefly speaking, we provide the solution for Question 2, in which the delivery of $c_{k}$ completes our transmission of method I and $X_{p}^{[k]}$ is transmitted based on method II.

For block 5, the transmission signals at relays are:

$$
\begin{aligned}
& x_{R_{1}}(5)=a_{1}\left(\sqrt{P^{\alpha}} F_{1}(2)^{T}\left[\begin{array}{l}
x_{p, 1}^{[1]} \\
x_{p, 1}^{[2]}
\end{array}\right]+z_{1}(2)\right), \\
& x_{R_{2}}(5)=\sqrt{P-P^{\alpha}} c_{1}+a_{2}\left(\sqrt{P^{\alpha}} F_{2}^{T}(2)\left[\begin{array}{c}
x_{p, 1}^{[1]} \\
x_{p, 1}^{[2]}
\end{array}\right]+z_{2}(2)\right), \\
& x_{R_{3}}(5)=a_{3}\left(\sqrt{P^{\alpha}} F_{3}^{T}(2)\left[\begin{array}{c}
x_{p, 1}^{[1]} \\
x_{p, 1}^{[2]}
\end{array}\right]+z_{3}(2)\right)
\end{aligned}
$$


where $a_{i}(i \in\{1,2,3\})$ are the amplification factors with the constraint $\sum_{i=1}^{3}\left|a_{i}\right|^{2}=1$. Then, the received signals at the destinations can be written as (omitting block index):

$$
\begin{aligned}
y_{1}= & \sqrt{P-P^{\alpha}} g_{12} c_{1} \\
& +\sqrt{P^{\alpha}}\left(\sum_{i=1}^{3} g_{1 i} a_{i} f_{i 1} x_{p, 1}^{[1]}+\sum_{i=1}^{3} g_{1 i} a_{i} f_{i 2} x_{p, 1}^{[2]}\right)+n_{1}^{\prime}, \\
y_{2}= & \sqrt{P-P^{\alpha}} g_{22} c_{1} \\
& +\sqrt{P^{\alpha}}\left(\sum_{i=1}^{3} g_{2 i} a_{i} f_{i 2} x_{p, 1}^{[2]}+\sum_{i=1}^{3} g_{2 i} a_{i} f_{i 1} x_{p, 1}^{[1]}\right)+n_{2}^{\prime},
\end{aligned}
$$

where $n_{k}^{\prime}=n_{k}+\sum_{i=3}^{3} g_{k i} a_{i} z_{i}$ represents the effective noise at $\mathcal{D}_{k}, k=1,2$. Apparently, $\mathbb{E}\left(\left|n_{k}^{\prime}\right|^{2}\right)=o(\log (P))$. The amplification factors are chosen to neutralize $x_{p, 1}^{[2]}$ and $x_{p, 1}^{[1]}$ at $\mathcal{D}_{1}$ and $\mathcal{D}_{2}$, respectively. Based on the instantaneous CSI, $a_{i}(i \in\{1,2,3\})$ are chosen such that

$$
\sum_{i=1}^{3} \hat{g}_{1 i} a_{i} f_{i 2}=0, \quad \sum_{i=1}^{3} \hat{g}_{2 i} a_{i} f_{i 1}=0 .
$$

Meanwhile, we can show $\sum_{i=1}^{3} g_{1 i} a_{i} f_{i 1}$ and $\sum_{i=1}^{3} g_{2 i} a_{i} f_{i 2}$ are non-zero almost surely. Consequently, the received signals at destinations can be re-written as follows

$$
\begin{aligned}
y_{1} & =\sqrt{P-P^{\alpha}} g_{12} c_{1} \\
& +\sqrt{P^{\alpha}}\left(\sum_{i=1}^{3} g_{1 i} a_{i} f_{i 1} x_{p, 1}^{[1]}+\sum_{i=1}^{3} \tilde{g}_{1 i} a_{i} f_{i 2} x_{p, 1}^{[2]}\right)+n_{1}^{\prime}, \\
y_{2} & =\sqrt{P-P^{\alpha}} g_{12} c_{1} \\
& +\sqrt{P^{\alpha}}\left(\sum_{i=1}^{3} g_{2 i} a_{i} f_{i 2} x_{p, 1}^{[2]}+\sum_{i=1}^{3} \tilde{g}_{2 i} a_{i} f_{i 1} x_{p, 1}^{[1]}\right)+n_{2}^{\prime} .
\end{aligned}
$$

We observe that three classes of information exist in the received signals, i.e., at $\mathcal{D}_{k}$, the received signal $y_{k}$ consists of the common message $c_{1}$, the extra new private message $x_{p, 1}^{[k]}$ and the interference leakage $x_{p, 1}^{[j]}$, for $j \neq k$. The desired message $c_{1}$ and $x_{p, 1}^{[k]}$ will be decoded successively.

Following a similar structure, the transmission signals in the block $b=6$ are

$$
\begin{aligned}
& x_{R_{1}}(6)=\sqrt{P-P^{\alpha}} c_{2}+b_{1}\left(\sqrt{P^{\alpha}} F_{1}^{T}(3)\left[\begin{array}{c}
x_{p, 2}^{[1]} \\
x_{p, 2}^{[2]}
\end{array}\right]+z_{1}(3)\right), \\
& x_{R_{2}}(6)=b_{2}\left(\sqrt{P^{\alpha}} F_{2}^{T}(3)\left[\begin{array}{l}
x_{p, 2}^{[1]} \\
x_{p, 2}^{[2]}
\end{array}\right]+z_{2}(3)\right), \\
& x_{R_{3}}(6)=b_{3}\left(\sqrt{P^{\alpha}} F_{3}^{T}(3)\left[\begin{array}{l}
x_{p, 2}^{[1]} \\
x_{p, 2}^{[2]}
\end{array}\right]+z_{3}(3)\right),
\end{aligned}
$$

where $b_{i}$ are chosen to satisfy a similar neutralization conditions accordingly as shown in (29). The details are omitted to avoid repetition.

Decoding at destinations: The decoding process consists of the following steps.

- Decode $c_{k}$ : Both destinations decode $c_{1}$ and $c_{2}$ from the received signals. By treating $X_{p}^{[k]}$ as noise, the SINR is on the level of $O\left(\frac{P-P^{\alpha}}{P^{\alpha}}\right)=O\left(P^{1-\alpha}\right)$. Then, the following rate can be achieved for decoding $c_{k}$ :

$$
R_{c}=(1-\alpha) \log (P)+o(\log (P)) .
$$

- Recover $\hat{\Sigma}_{k}$ from $c_{k}$ : The recovery of $\hat{\Sigma}_{i}$ is possible if $R_{\sigma_{k}} \leq R_{c}$. The proper $R_{\sigma_{k}}$ can be chosen to satisfy the above constraint.

- Decode $X_{p}^{[k]}$ : Destinations remove $c_{1}$ and $c_{2}$ from the received signals. For instance, at $\mathcal{D}_{1}$, after subtracting $c_{1}$ from (30), the residual signal can be represented as:

$$
\tilde{y}_{1}=\sqrt{P^{\alpha}}\left(\sum_{i=1}^{3} g_{1 i} a_{i} f_{i 1} x_{p, 1}^{[1]}+\sum_{i=1}^{3} \tilde{g}_{1 i} a_{i} f_{i 2} x_{p, 1}^{[2]}\right)+n_{1}^{\prime} \text {. }
$$

To decode $x_{p, 1}^{[1]}$, we can lower-bound the rate $R_{p 1}^{[1]}$ shown on the top of the next page. Let $\mathcal{H}$ represent the set of channel coefficients known at destinations. (35) follows from Jensen's Inequality, (36) follows from $\mathbb{E}_{H}\left(\left|\sum_{i=1}^{3} \tilde{g}_{1 i} a_{i} f_{i 2}\right|^{2}\right) \leq$ $\mathbb{E}_{H}\left(\left|\sum_{i=1}^{3} \tilde{g}_{1 i} f_{i 2}\right|^{2}\right)$ because of $\sum_{i=1}^{3}\left|a_{i}\right|^{2}=1$, and (37) follows from $\mathbb{E}\left(\tilde{g}_{1 i}^{2}\right)=\delta^{2}=P^{-\alpha}$ and the statistical independence of $f_{i j}$ and $g_{m n}$. Similarly, it is readily shown $R_{p 2}^{[1]}=\alpha \log (P)+o(\log (P))$. Therefore, $R_{p}^{[1]}=$ $2 \alpha \log (P)+o(\log (P))$. For decoding $X_{p}^{[2]}$, we also have $R_{p}^{[2]}=2 \alpha \log (P)+o(\log (P))$ from a similar approach.

- Decode $X_{o}^{[k]}$ : By collecting the received signals from two phases, $\mathcal{D}_{k}$ can decode $X_{o}^{[k]}$ by constituting an equivalent MIMO channel as shown in Appendix B with rate:

$$
R_{o}^{[k]}=(2-\alpha) \log (P)+o(\log (P)) .
$$

Combining the rate constraints of two hops, the following sum DOF is achieved

$$
d=\lim _{P \rightarrow \infty} \frac{R_{1}+R_{2}}{3 \log (P)}=\frac{2(2-\alpha+2 \alpha)}{3}=\frac{4+2 \alpha}{3} .
$$

Remark 2: Note that in block $b=5$, we manage to convey the common message $c_{1}$ with the DOF $1-\alpha$, and the private messages $x_{p, 1}^{[k]}$ to $\mathcal{D}_{k}$ with DOF $\alpha$, for $k=1,2$. This observation directly show that the DOF pairs $(\alpha, 1)$ and $(1, \alpha)$ can be achieved by simply absorbing the common message into either private message. Therefore, the DOF region (6) can be settled as optimal by time sharing among all the vertices.

We finish the proof here.

\section{Discussions}

\section{A. Generalizations to Multi-hop and Multi-antenna Networks}

The result shown in Theorem 1 is not limited to the twohop case. We now consider a more general $N$-hop $(N \geq 2)$ system with a 2 -antenna source, 2 single-antenna destinations, and $N-1$ layers of single-antenna relays in between. No physical connection exists between terminals in non-adjacent layers. We treat the source as layer-0. Let $I_{n}$ represent the number of relays at layer- $n$ for $n \in\{1,2, \cdots, N-1\}$. Mixed CSIT is available but limited within each hop. We term this network as the $N$-hop $2 \times I^{N-1} \times 2$ MISO broadcast network. Its sum DOF is presented in the following corollary.

Corollary 1: With mixed CSIT and at least 3 relays in each relay layer, the considered $N$-hop $2 \times I^{N-1} \times 2$ MISO broadcast network has sum optimal DOF $\frac{4+2 \alpha}{3}$, for $0 \leq \alpha \leq 1$. 


$$
\begin{aligned}
R_{p 1}^{[1]}= & I\left(x_{p, 1}^{[1]} ; \tilde{y}_{1}, \mathcal{H}\right)=\mathbb{E}_{H}\left[\log \left(1+\frac{P^{\alpha}\left|\sum_{i=1}^{3} g_{1 i} a_{i} f_{i 1}\right|^{2}}{o(\log (P))+P^{\alpha}\left|\sum_{i=1}^{3} \tilde{g}_{1 i} a_{i} f_{i 2}\right|^{2}}\right)\right] \\
\geq & \mathbb{E}_{H}\left\{\log \left[P^{\alpha}\left(\left|\sum_{i=1}^{3} g_{1 i} a_{i} f_{i 1}\right|^{2}+\left.\sum_{i=1}^{3} \tilde{g}_{1 i} a_{i} f_{i 2}\right|^{2}\right)+o(\log (P))\right]\right\} \\
& \quad-\log \left(P^{\alpha} \mathbb{E}_{H}\left(\left|\sum_{i=1}^{3} \tilde{g}_{1 i} a_{i} f_{i 2}\right|^{2}\right)+o(\log (P))\right) \\
\geq & \mathbb{E}_{H}\left(\log \left(P^{\alpha}\left|\sum_{i=1}^{3} g_{1 i} a_{i} f_{i 1}\right|^{2}\right)\right)-\log \left(P^{\alpha} \mathbb{E}_{H}\left(\left|\sum_{i=1}^{3} \tilde{g}_{1 i} f_{i 2}\right|^{2}\right)+o(\log (P))\right) \\
= & \alpha \log (P)+o(\log (P))-o(\log (P))=\alpha \log (P)+o(\log (P))
\end{aligned}
$$

Proof: The converse follows directly from Section IV-A. For achievability, we start with $N=3$ and $I_{1}=I_{2}=3$ (the 3hop network). Let $\mathcal{R}_{i}^{[n]}(i \in\{1,2,3\}$ and $n \in\{1,2\})$ represent the $i$-th relay in layer- $n$. Keep the rate splitting and encoding procedures the same as the achievability proof of Theorem 1 . The transmission still works in the two-phase manner. Let us focus on the layer-2 at first. We observe that if $\mathcal{R}_{k}^{[2]}$ can decode $X_{o}^{[k]}$ for $k \in\{1,2\}$, then by the assist of the received signal at $\mathcal{R}_{3}^{[2]}$ the similar beamforming scheme can be designed in this layer as shown in Fig. 3. Furthermore, if $\mathcal{R}_{k}^{[2]}$ does not intend to decode $X_{p}^{[k]}$ but simply amplifies and forwards it to destinations, then the amplification factors can be designed similar to the neutralization requirements (29). In this way, $\mathcal{R}_{i}^{[2]}(i \in\{1,2,3\})$ plays the same role as $\mathcal{R}_{i}$ in the two-hop network. Consequently, the same DOF can be achieved in this 3 -hop network. To achieve this goal, we first consider the layer 0,1 and 2 as a subnetwork. We simply treat $\mathcal{R}_{1}^{[2]}$ and $\mathcal{R}_{2}^{[2]}$ as the virtue destinations to decode $X_{o}^{[1]}$ and $X_{o}^{[2]}$, respectively. Meanwhile, set the amplification factor at $\mathcal{R}_{i}^{[1]}$ to be 1 . Then, after the phase two at the second hop, we consider the layer 2 and 3 as a new subnetwork. We can design the transmission from layer 2 to 3 similar to the approach introduced in Section IV-E. Clearly, by this recursive approach, the same DOF $\frac{4+2 \alpha}{3}$ can be achieved. By induction, for $N \geq 3$, the achievability follows a similar approach to obtain the sum DOF $\frac{4+2 \alpha}{3}$.

Remark 3: Corollary 1 shows an interesting result: In a wireless network with a two-antenna source and two singleantenna destinations, implementing multiple hops of distributed processing does not reduce network DOF, as long as the number of relays at each layer is not less than 3 .

In addition, we consider a network consisting of a $2 M$ antenna source, two $M$-antenna destinations, and $I(I \geq 3)$ $M$-antenna relays in between. We term this network as the 2-hop $2 M \times I M \times 2 M$ MIMO broadcast network. The DOF results can be directly generalized to the MIMO case, where the achievable scheme is the matrix form accordingly.

Corollary 2: With mixed CSIT and $I \geq 3$ relays, the considered 2-hop $2 M \times I M \times 2 M$ MIMO broadcast network has sum optimal DOF $\frac{(4+2 \alpha) M}{3}$, for $0 \leq \alpha \leq 1$.

\section{B. Discussions on the Half-Duplex Relay Networks}

It is observed that the achievablity throughout the paper is based on full-duplex relaying. It is interesting to note that the sum DOF $\frac{4+2 \alpha}{3}$ can also be achieved by half-duplex relaying, if the number of relays is not less than 6 . The key idea is to apply cluster successive relaying [29] to the considered network. We provide the sketch of the achievability as follows. The relays can be divided into two disjoint sets, say $\mathcal{A}_{1}$ and $\mathcal{A}_{2}$. The cardinality of each set is not less than 3. Accordingly, we can divide the message into two parts, each of which will be transmitted through different relay set. Then, adopting the principle of successive relaying, the relays in set $\mathcal{A}_{1}$ can work orthogonally to the relays in set $\mathcal{A}_{2}$ for receiving and transmitting. Equivalently, these two orthogonal sets of relays can be seen as one set of relays which can receive and transmit signals at the same time. Therefore, by twice number of relays, we can design two groups of communications orthogonally to mimic the behavior of full-duplex relaying. The same DOF $\frac{4+2 \alpha}{3}$ can be achieved.

\section{The Polyhedron Structure of DOF Region with Common Message}

The main result can be extended directly to the case with common message.

Corollary 3: Let $\left(d_{0}, d_{1}, d_{2}\right)$ represent the DOF of the common message, private message for $D_{1}$, and private message for $D_{2}$, respectively. Then, the optimal DOF region is characterized by

$$
\begin{aligned}
d_{0}+d_{1} & \leq 1, \\
d_{0}+d_{2} & \leq 1, \\
2 d_{0}+d_{1}+2 d_{2} & \leq 2+\alpha, \\
2 d_{0}+2 d_{1}+d_{2} & \leq 2+\alpha,
\end{aligned}
$$

for the $2 \times I \times 2$ network with mixed CSIT when $I \geq 3$.

Proof: The converse follows quickly by the same mathod as proposed in the upper bound proof of Theorem 1. The DOF region outer bounds for the enhanced second hop (fully cooperated relays with source messages) are the outer bounds for our considered two-hop network, with details shown in [11] and [12]. 


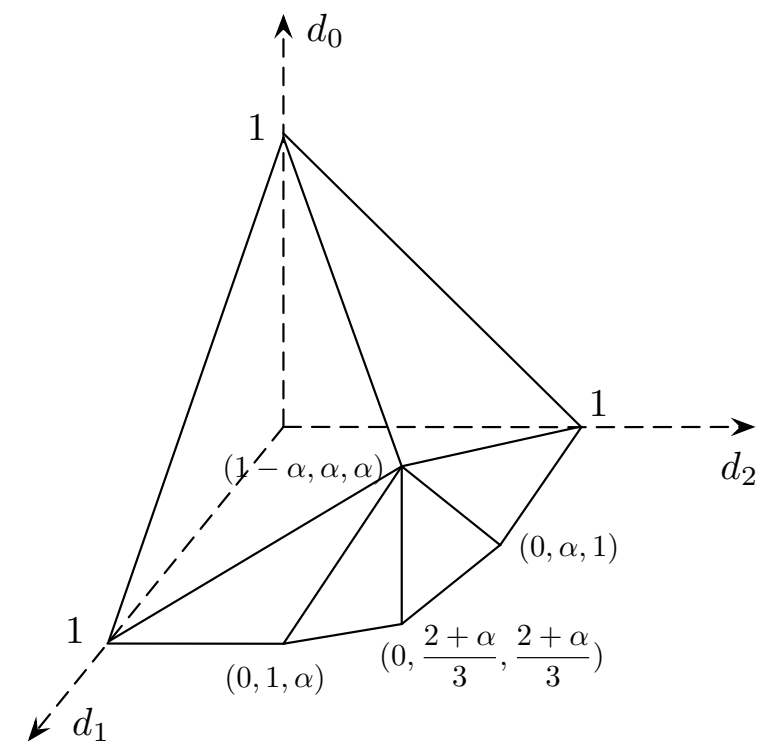

Fig. 5. The DOF polyhedron with common messages.

The achievability is shown by settling the following vertices of the DOF polyhedron as shown in Fig. 5 and time sharing, where the achievability of vertices is also implied by the proof of Theorem 1 in Section IV-D and IV-E.

\section{Discussions on the Sum DOF of the $2 \times 2 \times 2$ Network}

The sum DOF of the $2 \times 2 \times 2$ network with mixed CSIT still remains open. We summaize the best known lower and upper bound for the sum DOF as follows:

$$
\max \left\{\frac{4}{3}, 2 \alpha\right\} \leq d \leq \frac{4+2 \alpha}{3} .
$$

The upper bound comes directly from Theorem 1. For the lower bound, $\frac{4}{3}$ is clearly achieved only based on the delayed CSIT as shown in Lemma 1 , while $2 \alpha$ is achieved only based on the instantaneous CSIT shown in Proposition 1 (please refer to Appendix C). However, the question remains that if the combination of both forms of CSIT can bring extra benefit to DOF.

\section{CONCLUSIONS}

The sum DOF of the two-hop MISO broadcast network consisting of a two-antenna source and two destinations with multiple single-antenna relays has been studied. We consider scenarios with mixed CSIT, which consists of the two kinds of CSIT, namely, perfect delayed and inaccurate instantaneous. When the number of relays is 3 or more, we settle the sum DOF of the considered network to be the same as the singlehop broadcast channel, showing that the multi-hop network structure and distributed processing at relays do not degrade DOF. Our results are the first to show that the benefits of these two categories of CSIT can be accumulated in a multihop network scenario. The explicit DOF establishment for networks with 2 relays remains as an open problem. We also note that throughout the paper, the instantaneous CSIT uncertainty is assumed to lie on the same level for both hops.
However, in practice, due to different correlation scenarios of individual hops, the correlation factors will affect the CSIT uncertainty of each hop independently. In this scenario, the impact of the correlation factors on the DOF of multihop networks is an interesting and meaningful research topic.

\section{APPENDIX A \\ PROOF OF EQUATION (25)}

We consider the average power of interference with respect to logarithm. For instance, when $k=1$, we have $\mathbb{E}\left(\log \left(\left|\sigma_{1}\right|^{2}\right)\right)=\mathbb{E}_{H, X}\left(\log \left(\left|\sigma_{1}\right|^{2}\right)\right) \leq \mathbb{E}_{H}\left(\log \left(\mathbb{E}_{X}\left(\left|\sigma_{1}\right|^{2}\right)\right)\right)$, followed by Jensen's inequality. Define $\left|\eta_{1}\right|^{2}=\mathbb{E}_{X}\left(\left|\sigma_{1}\right|^{2}\right)$. Then,

$$
\begin{aligned}
\left|\eta_{1}\right|^{2}= & {\left[\begin{array}{ll}
\frac{g_{12}}{\hat{g}_{12}} & \tilde{g}_{13}-\frac{\tilde{g}_{12}}{\hat{g}_{12}} \hat{g}_{13}
\end{array}\right]\left[\begin{array}{cc}
Q_{1} Q_{1}^{H} & 0 \\
0 & L_{2} L_{2}^{H}
\end{array}\right] } \\
& {\left[\begin{array}{ll}
\frac{g_{12}}{\hat{g}_{12}} & \tilde{g}_{13}-\frac{\tilde{g}_{12}}{\hat{g}_{12}} \hat{g}_{13}
\end{array}\right]^{H} } \\
= & \left|\frac{g_{12}}{\hat{g}_{12}}\right|^{2} P^{1-\alpha}+\left|\tilde{g}_{13}-\frac{\tilde{g}_{12}}{\hat{g}_{12}} \hat{g}_{13}\right|^{2} L_{2} L_{2}^{H} .
\end{aligned}
$$

As we can see, if the second moment of $\eta_{1}$ with respect to the logarithm is approximately $(1-\alpha) \log (P)$, by the ratedistortion theorem [29] it is equivalent to have the average power $P^{1-\alpha}$. In what follows, we adopt this alternative approach. Averaging the logarithm of $\left|\eta_{1}\right|^{2}$ over channel realizations:

$$
\begin{aligned}
\mathbb{E}_{H}\left(\log \left(\left|\eta_{1}\right|^{2}\right)\right)= & \mathbb{E}_{H}\left(\log \left(P^{1-\alpha}+L_{2} L_{2}^{H}\left|\hat{g}_{12} \tilde{g}_{13}-\tilde{g}_{12} \hat{g}_{13}\right|^{2}\right)\right) \\
& \quad-\mathbb{E}_{H}\left(\log \left(\hat{g}_{12}^{2}\right)\right) \\
& \stackrel{(a)}{\leq} \log \left(P^{1-\alpha}+P \mathbb{E}_{H}\left(\left|\hat{g}_{12} \tilde{g}_{13}-\tilde{g}_{12} \hat{g}_{13}\right|^{2}\right)\right) \\
& \quad-\mathbb{E}_{H}\left(\log \left(\hat{g}_{12}^{2}\right)\right) \\
& \stackrel{(b)}{=}(1-\alpha) \log (P)+o(\log (P))-\mathbb{E}_{H}\left(\log \left(\hat{g}_{12}^{2}\right)\right),
\end{aligned}
$$

where $(a)$ follows from Jensen's inequality and $\mathbb{E}_{H}\left(L_{2} L_{2}^{H}\right)=$ $P$, and $(b)$ follows from $\mathbb{E}_{H}\left(\left|\hat{g}_{12} \tilde{g}_{13}-\tilde{g}_{12} \hat{g}_{13}\right|^{2}\right)=O\left(P^{-\alpha}\right)$. Moreover, we can show that $\mathbb{E}_{H}\left(\log \left(\hat{g}_{12}^{2}\right)\right)=\log \left(1-P^{-\alpha}\right)+$ $O(1)$ [30], which indicates $\mathbb{E}_{H}\left(\log \left(\hat{g}_{12}^{2}\right)\right)=o(\log (P))$.

Therefore, we have

$$
\mathbb{E}_{H}\left(\log \left(\left|\eta_{1}\right|^{2}\right)\right)=(1-\alpha) \log (P)+o(\log (P)) .
$$

Similarly, for $k=2$ it follows the same approach. We finish the proof of (25).

\section{APPENDIX B
PRoOF OF INEQUALITY (34): RATE OF $X_{O}^{[k]}$}

Because of symmetry, we only consider the rate of $X_{O}^{[1]}$. After the decoding of common messages $c_{1}$ and $c_{2}$, the quantized sequence $\hat{\Sigma}_{1}$ and $\hat{\Sigma}_{2}$ can be recovered by destinations. Let $\Delta_{k}(k \in\{1,2\})$ represent the quantization noise, thus we have $\Sigma_{k}=\hat{\Sigma}_{k}+\Delta_{k}$, where $\Delta_{k}$ is the quantization noise with average distortion $D_{k}=\mathbb{E}\left(\left|\Delta_{k}\right|^{2}\right)=1$. For $\mathcal{D}_{1}$, the following MIMO channel can be constructed by collecting the received signal during $b=4$ (19) and $\hat{\Sigma}_{k}$ :

$$
\bar{Y}_{1}=\left[\begin{array}{c}
\hat{\Sigma}_{2} \\
y_{1}(4)-\hat{\Sigma}_{1}
\end{array}\right]=\mathbf{S} X_{o}^{[1]}+\left[\begin{array}{c}
-\Delta_{2} \\
n_{1}+\Delta_{1}
\end{array}\right]
$$


where

$$
\mathbf{S}=\underbrace{\left[\begin{array}{cc}
\frac{g_{21}}{\hat{g}_{21}} & \tilde{g}_{23}-\frac{\tilde{g}_{21}}{\hat{g}_{21}} \hat{g}_{23} \\
\frac{g_{11}}{\hat{g}_{21}} & g_{13}-\frac{g_{11}}{\hat{g}_{21}} \hat{g}_{23}
\end{array}\right]}_{\mathbf{G}}\left[\begin{array}{c}
Q_{2} \\
L_{1}
\end{array}\right] .
$$

We can lower-bound $R_{o}^{[1]}$ as follows:

$$
\begin{aligned}
& R_{o}^{[1]}=I\left(X_{o}^{[1]} ; \bar{Y}_{1} \mid \mathbf{S}\right)=I\left(\mathbf{S} X_{o}^{[1]} ; \bar{Y}_{1}\right) \\
& =H\left(\mathbf{S} X_{o}^{[1]}\right)-H\left(\left[\begin{array}{c}
-\Delta_{2} \\
n_{1}+\Delta_{1}
\end{array}\right] \mid \bar{Y}_{1}\right) \\
& \geq H\left(\mathbf{S} X_{o}^{[1]}\right)-\log \left(1+D_{1}\right)-\log \left(D_{2}\right) \\
& =\mathbb{E}_{H}\left(\log \left|\mathbf{G G}^{H}\right|\right)+\mathbb{E}_{H}\left(\log \left|\begin{array}{cc}
Q_{2} Q_{2}^{H} & 0 \\
0 & L_{1} L_{1}^{H}
\end{array}\right|\right) \\
& +o(\log (P)) \\
& =(1-\alpha) \log (P)+\mathbb{E}_{H}\left(\log \left|\mathbf{G G}^{H}\right|\right)+\mathbb{E}_{H}\left(\log \left|L_{1} L_{1}^{H}\right|\right) \\
& +o(\log (P))
\end{aligned}
$$

(41) follows from that $\mathbf{S}$ is invertible almost surely (which can be readily shown through $|\mathbf{S}| \neq 0$ with probability one) and thus the linear transformation is information-lossless; (42) follows from that conditioning does not increase differential entropy and Gaussian distribution maximize the entropy of the distortion term; (43) follows from $D_{1}=D_{2}=1$ and $Q_{2} \perp L_{1}$; (44) follows simply by substituting $\left\|Q_{2}\right\|_{2}^{2}=P^{1-\alpha}$ in it. To proceed, in (44) the term $\mathbb{E}_{H}\left(\log \left|L_{1} L_{1}^{H}\right|\right)$ can be bounded as follows:

$$
\begin{aligned}
\mathbb{E}_{H}\left(\log \left|L_{1} L_{1}^{H}\right|\right) & =\mathbb{E}_{H}\left(\log \left|F_{3}^{H} \mathbf{V}_{1} \mathbf{P} \mathbf{V}_{1}^{H} F_{3}\right|\right) \\
& \geq \mathbb{E}_{H}\left(\log \left|\left(P-P^{1-\alpha}\right) F_{3}^{H} V_{11} V_{11}^{H} F_{3}\right|\right) \\
& =\log (P)+o(\log (P)) .
\end{aligned}
$$

The next step is to show $\mathbb{E}_{H}\left(\log \left|\mathbf{G G}^{H}\right|\right) \geq o(\log (P))$, with the matrix $\mathbf{G}$ defined in (40). Let us introduce Hadamard product denoted by $\circ$. Then, $\mathbf{G}$ can be written as $\mathbf{G}=\mathbf{G}_{1} \circ \mathbf{G}_{2}$, with $\mathbf{G}_{1}$ and $\mathbf{G}_{2}$ defined as follows:

$$
\mathbf{G}_{1}=\left[\begin{array}{ll}
g_{21} & 1 \\
g_{11} & 1
\end{array}\right] \quad \mathbf{G}_{2}=\left[\begin{array}{cc}
\frac{1}{\hat{g}_{21}} & \tilde{g}_{23}-\tilde{g}_{21} \frac{\hat{g}_{23}}{\hat{g}_{21}} \\
\frac{1}{\hat{g}_{21}} & g_{13}-g_{11} \frac{g_{23}}{\hat{g}_{21}}
\end{array}\right] .
$$

By Schur product theorem, we have $|\mathbf{G}| \geq\left|\mathbf{G}_{1}\right|\left|\mathbf{G}_{2}\right|$. Then,

$$
\begin{aligned}
\mathbb{E}_{H}\left(\log \left|\mathbf{G G}^{H}\right|\right) \geq & \mathbb{E}_{H}\left(\log \left|\mathbf{G}_{1}\right|^{2}\right)+\mathbb{E}_{H}\left(\log \left|\mathbf{G}_{2}\right|^{2}\right) \\
= & o(\log (P))+\mathbb{E}_{H}\left(\log \left|\mathbf{G}_{2}\right|^{2}\right) \\
= & o(\log (P))+\mathbb{E}_{H}\left(\log |\rho|^{2}\right) \\
& -2 \mathbb{E}_{H}\left(\log \left|\hat{g}_{21}\right|^{2}\right)
\end{aligned}
$$

where (46) follows from the the determinant of $\mathbf{G}_{1}$ which is a constant with respect to $P$; (47) follows from the calculation of the determinant of $\mathbf{G}_{2}$, where $\rho=\left(g_{13}-\tilde{g}_{23}\right) \hat{g}_{21}+\left(\tilde{g}_{21}-\right.$ $\left.g_{11}\right) \hat{g}_{23}$. Following the principle of the algebra of random variables, it can be shown that $\rho$ has variance $2\left(1+\delta^{2}\right)\left(1-\delta^{2}\right)$. Therefore, we can obtain $\mathbb{E}_{H}\left(\log |\rho|^{2}\right)=O(1)+\log ((1+$ $\left.\left.\delta^{2}\right)\left(1-\delta^{2}\right)\right)=O(1)+\log \left(\left(1+P^{-\alpha}\right)\left(1-P^{-\alpha}\right)\right)=o(\log (P))$. Similar analysis can be applied for the third term in (47) to obtain $\mathbb{E}_{H}\left(\log \left|\hat{g}_{21}\right|^{2}\right)=o(\log (P))$. Therefore, back to (47), we have $\mathbb{E}_{H}\left(\log \left|\mathbf{G G}^{H}\right|\right) \geq o(\log (P))$. Therefore, back to (44), we have $R_{o}^{[1]} \geq(2-\alpha) \log (P)+o(\log (P))$. This concludes our proof.

\section{APPENDIX C}

THE ACHIEVED DOF OF LBF SCHEMES WITH INACCURATE INSTANTANEOUS CSIT

Proposition 1: With only inaccurate instantaneous CSIT as defined in Section II, for the $2 \times I \times 2$ broadcast network $(I \geq 2), 2 \alpha$ sum DOF can be achieved by LBF-based schemes.

Proof: It suffices to show that $2 \alpha$ DOF can be achieved for the $2 \times 2 \times 2$ network. Specifically, we apply the interference neutralization approach proposed in [20]. Then, the achieved DOF is analyzed based on the inaccuracy of CSIT.

We consider the $M$-symbol time-extension of the original channel. The channel input-output relationship is:

$$
\begin{aligned}
& Y_{R_{k}}(b, t)=\mathbf{F}_{k}(b, t) X(b, t)+Z_{k}(b, t), \\
Y_{k}(b, t)= & \mathbf{G}_{k 1}(b, t) X_{R_{1}}(b, t)+\mathbf{G}_{k 2}(b, t) X_{R_{2}}(b, t)+N_{k}(b, t),
\end{aligned}
$$

where $\mathbf{F}_{k}(b, t)=\left[\mathbf{F}_{k 1}(b, t) \mathbf{F}_{k 2}(b, t)\right]$, with $\mathbf{F}_{k i}(b, t)=$ $\operatorname{diag}\left\{f_{k i}(b,(M-1) t+m)\right\}$ and $\mathbf{G}_{k i}(b, t)=\operatorname{diag}\left\{g_{k i}(b,(M-\right.$ $1) t+m)\}$, for $m \in\{1,2, \ldots, M\} . X(b, t)$ is a $2 M \times 1$ vector representing the $M$-symbol extension at two antennas. Correspondingly, $X_{R_{1}}(b, t)$ and $X_{R_{2}}(b, t)$ are $M \times 1$ vectors. At the current time slot $(b, t)$, we represent the channel as $\mathbf{F}_{k i}(b, t)=$ $\hat{\mathbf{F}}_{k i}(b, t)+\tilde{\mathbf{F}}_{k i}(b, t)$, where $\hat{\mathbf{F}}_{k i}(b, t)=\operatorname{diag}\left\{\hat{f}_{k i}(b,(M-1) t+\right.$ $m)\}$ and $\tilde{\mathbf{F}}_{k i}(b, t)=\operatorname{diag}\left\{\tilde{f}_{k i}(b,(M-1) t+m)\right\}$ denote the estimated channel and the estimation error, respectively. Clearly, the covariance matrices of $\hat{\mathbf{F}}_{k i}(b, t)$ and $\tilde{\mathbf{F}}_{k i}(b, t)$ are $\left(1-\sigma^{2}\right) \mathbf{I}$ and $\sigma^{2} \mathbf{I}$, respectively. Similarly, $\hat{\mathbf{G}}_{k i}(b, t)$ and $\tilde{\mathbf{G}}_{k i}(b, t)$ follow the same extension. Again, for simplicity, we omit the time index $t$ in the indices pair $(b, t)$ hereafter.

Let the $M \times 1$ vector $X^{[k]}$ represent the codeword intended for $\mathcal{D}_{k}$. We transmit the symbols with power $P$, then the

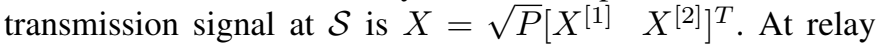
$\mathcal{R}_{i}, i \in\{1,2\}$, the transmitted signal is $X_{R_{i}}=\mathbf{A}_{i} Y_{R_{i}}$, where $\mathbf{A}_{i}$ is an $M \times M$ beamforming matrix. Thus, at destinations, we have

$$
\begin{aligned}
& Y_{1}=\sqrt{P}\left(\mathbf{H}_{11} X^{[1]}+\mathbf{H}_{12} X^{[2]}\right)+\mathbf{Z}_{1}^{\prime} \\
& Y_{2}=\sqrt{P}\left(\mathbf{H}_{21} X^{[1]}+\mathbf{H}_{22} X^{[2]}\right)+\mathbf{Z}_{2}^{\prime}
\end{aligned}
$$

where $\mathbf{H}_{k i}=\mathbf{G}_{k 1} \mathbf{A}_{1} \mathbf{F}_{1 i}+\mathbf{G}_{k 2} \mathbf{A}_{2} \mathbf{F}_{2 i}$ and $\mathbf{Z}_{k}^{\prime}=\mathbf{G}_{k 1} \mathbf{A}_{1} Z_{1}+$ $\mathbf{G}_{k 2} \mathbf{A}_{2} Z_{2}+N_{k}$. We design $\mathbf{A}_{1}$ and $\mathbf{A}_{2}$ based on the inaccurate $\hat{\mathbf{G}}_{k i}$ such that the interference matrices

$$
\begin{aligned}
\hat{\mathbf{H}}_{12} & =\hat{\mathbf{G}}_{11} \mathbf{A}_{1} \mathbf{F}_{12}+\hat{\mathbf{G}}_{12} \mathbf{A}_{2} \mathbf{F}_{12}=\Upsilon \Phi^{H} ; \\
\hat{\mathbf{H}}_{21} & =\hat{\mathbf{G}}_{21} \mathbf{A}_{1} \mathbf{F}_{11}+\hat{\mathbf{G}}_{22} \mathbf{A}_{2} \mathbf{F}_{11}=0
\end{aligned}
$$

where $\Upsilon$ and $\Phi$ are two $M \times 1$ vectors whose entries are drawn identically and independently from a continuous distribution with finite variance. Therefore, $\operatorname{rank}\left(\hat{\mathbf{H}}_{12}\right)=1$, indicating to null the interference in $M-1$ dimensions of its rangespace. The solution of $\mathbf{A}_{i}$ is given in [20] and we do not repeat here. Let $A_{i}(m, n)$ represent the $(m, n)$ th entry in $\mathbf{A}_{i}$. It is worth noting that $A_{i}(m, n)$ can be written as $A_{i}(m, n)=$ $\left(\sqrt{1-\sigma^{2}}\right)^{-1} \bar{A}_{i}(m, n)=\left(\sqrt{1-P^{-\alpha}}\right)^{-1} \bar{A}_{i}(m, n)$, where $\bar{A}_{i}(m, n)$ is a random variable which does not rely on $P$. This property will be applied when we calculate the covariance matrix of the interference. Let the $(M-1) \times M$ matrix $\boldsymbol{\Gamma}^{H}$ 
represent the zero-forcing matrix at $\mathcal{D}_{1}$, where $\boldsymbol{\Gamma}^{H} \boldsymbol{\Gamma}=\mathbf{I}$ and $\boldsymbol{\Gamma} \hat{\mathbf{H}}_{12}=\mathbf{0}$. Let $\bar{Y}_{1}=\boldsymbol{\Gamma}^{H} Y_{1}$,

$$
\bar{Y}_{1}=\sqrt{P}\left(\boldsymbol{\Gamma}^{H} \mathbf{H}_{11} X^{[1]}+\boldsymbol{\Gamma}^{H} \tilde{\mathbf{H}}_{12} X^{[2]}\right)+\boldsymbol{\Gamma}^{H} \mathbf{Z}_{1}^{\prime}
$$

where $\tilde{\mathbf{H}}_{k i}=\tilde{\mathbf{G}}_{k 1} \mathbf{A}_{1} \mathbf{F}_{1 i}+\tilde{\mathbf{G}}_{k 2} \mathbf{A}_{2} \mathbf{F}_{2 i}$. Let $\tilde{\mathbf{K}}_{12}=$ $\mathbb{E}\left(\tilde{\mathbf{H}}_{12} \tilde{\mathbf{H}}_{12}^{H}\right)$ and $\mathbf{K}_{Z}=\mathbb{E}\left(\mathbf{Z}_{1}^{\prime} \mathbf{Z}_{1}^{\prime H}\right)$ represent the covariance matrices. Then, we have $\tilde{\mathbf{K}}_{12}=\mathbb{E}\left(\tilde{\mathbf{H}}_{12} \tilde{\mathbf{H}}_{12}^{H}\right)=$ $\mathbb{E}\left(\tilde{\mathbf{G}}_{11} \mathbf{K}_{1} \tilde{\mathbf{G}}_{11}^{H}+\tilde{\mathbf{G}}_{12} \mathbf{K}_{2} \tilde{\mathbf{G}}_{12}^{H}\right)=O\left(P^{-\alpha}\right) \mathbf{I}$, with $\mathbf{K}_{i}=$ $\mathbf{A}_{i} \mathbf{F}_{12} \mathbf{F}_{12}^{H} \mathbf{A}_{i}^{H}, i \in\{1,2\}$. It follows from the independence of $\tilde{\mathbf{G}}_{1 i}$ and $\mathbf{K}_{i}$, and the covariance matrix of $\tilde{\mathbf{G}}_{1 i}$ is $O\left(P^{-\alpha}\right) \mathbf{I}$. Similarly, we can readily show $\mathbf{K}_{Z}=O(1) \overline{\mathbf{K}}_{Z}$, where every entry of $\overline{\mathbf{K}}_{Z}$ does not rely on $P$. Let $R_{p}^{[i]}$ denote the rate of $X^{[i]}$. For $i=1$,

$$
\begin{aligned}
R_{p}^{[1]}= & I\left(X^{[1]} ; \bar{Y}_{1}, \mathcal{H}\right) \\
= & \mathbb{E}\left(\log \left|\bar{Y}_{1} \bar{Y}_{1}^{H}\right|\right)-\mathbb{E}\left(\log \left|\boldsymbol{\Gamma}^{H}\left(P \tilde{\mathbf{H}}_{12} \tilde{\mathbf{H}}_{12}^{H}+\mathbf{Z}_{1}^{\prime} \mathbf{Z}_{1}^{\prime}\right) \boldsymbol{\Gamma}\right|\right) \\
\geq & \mathbb{E}\left(\log \left|P \boldsymbol{\Gamma}^{H} \mathbf{H}_{11} \mathbf{H}_{11}^{H} \boldsymbol{\Gamma}\right|\right) \\
& -\log \left|\mathbb{E}\left(\boldsymbol{\Gamma}^{H}\left(P \tilde{\mathbf{H}}_{12} \tilde{\mathbf{H}}_{12}^{H}+\mathbf{Z}_{1}^{\prime} \mathbf{Z}_{1}^{\prime}\right) \boldsymbol{\Gamma}\right)\right| \\
= & \log (P)+\mathbb{E}\left(\log \left|\boldsymbol{\Gamma}^{H} \mathbf{H}_{11} \mathbf{H}_{11}^{H} \boldsymbol{\Gamma}\right|\right) \\
& -\log \left|\boldsymbol{\Gamma}^{H}\left(P \tilde{\mathbf{K}}_{12}+\mathbf{K}_{Z}\right) \boldsymbol{\Gamma}\right| \\
= & (M-1) \alpha \log (P)+o(\log (P))
\end{aligned}
$$

where (55) follows from Jensen's inequality and $\left|\bar{Y}_{1} \bar{Y}_{1}^{H}\right| \geq$ $\left|P \boldsymbol{\Gamma}^{H} \mathbf{H}_{11} \mathbf{H}_{11}^{H} \boldsymbol{\Gamma}\right|$, and (56) follows from $P \tilde{\mathbf{K}}_{12}+\mathbf{K}_{Z}$ is dominated by $P \tilde{\mathbf{K}}_{12}$ and $P \tilde{\mathbf{K}}_{12}=O\left(P^{1-\alpha}\right) \mathbf{I}$. Analogously, we can prove $X^{[2]}$ can be decoded at the rate $R_{p}^{[2]}=\alpha M \log (P)+$ $o(\log (P))$. Therefore, the sum DOF $\frac{2 M-1}{M} \alpha$ can be achieved. When $M \rightarrow \infty, 2 \alpha$ can be achieved asymptotically.

For $I \geq 3$, the scalar amplify-forward relaying can achieve the sum DOF $2 \alpha$ without symbols extension, based on the method shown in (7) and (8). To avoid repetition, we omit the proof here.

\section{REFERENCES}

[1] G. Caire and S. Shamai, "On the achievable throughput of a multiantenna Gaussian broadcast channel," IEEE Trans. Inform. Theory, vol. 49, pp. 1691-1706, Jul. 2003.

[2] J. Lee and N. Jindal, "High SNR analysis for MIMO broadcast channels: dirty paper coding versus linear precoding," IEEE Trans. Inform. Theory, vol. 53, pp. 4787-4792, Dec. 2007.

[3] G. Caire, N. Jindal, M. Kobayashi, and N. Ravindran, "Multiuser MIMO achievable rates with downlink training and channel state feedback," IEEE Trans. Inform. Theory, vol. 56, pp. 2845-2866, Jun. 2010.

[4] M. A. Maddah-Ali and D. Tse, "Completely stale transmitter channel state information is still very useful," IEEE Trans. Inform. Theory, vol. 58, pp. 4418-4431, Jul. 2012.

[5] N. Jindal, "MIMO broadcast channels with finite-rate feedback," IEEE Trans. Inform. Theory, vol. 52, pp. 5045-5060, Nov. 2006.

[6] J. Xu, J. G. Andrews, and S. A. Jafar, "MISO broadcast channels with delayed finite-rate feedback: predict or observe?" IEEE Trans. Wireless Commun., vol. 11, pp. 1456-1467, Apr. 2012.

[7] Z. Wang, C. Wang, M. Xiao, and M. Skoglund, "The two-hop MISO broadcast network with quantized delayed CSIT," in IEEE Wireless Commun. and Networking Conference (WCNC), Shanghai, China, Apr 2013.

[8] H. Maleki, S. A. Jafar, and S. Shamai, "Retrospective interference alignment," in Proc. IEEE Int. Symp. on Inf. Theory (ISIT), St. Petersburg, Jul. 2011, pp. 2756-2760.

[9] S. A. Jafar and A. J. Goldsmith, "Isotropic fading vector broadcast channels: the scalar upper bound and loss in degrees of freedom," IEEE Trans. Inform. Theory, vol. 51, no. 3, pp. 848-857, Mar. 2005.
[10] M. Kobayashi, S. Yang, D. Gesbert, and X. Yi, "On the degrees of freedom of time correlated MISO broadcast channel with delayed CSIT," in Proc. IEEE ISIT, Boston, July 2012.

[11] S. Yang, M. kobayashi, D. Gesbert, and X. Yi, "Degrees of freesom of time correlated MISO broadcast channel with delayed CSIT," IEEE Trans. Inform. Theory, vol. 59, pp. 315-328, Jan. 2013.

[12] T. Gou and S. A. Jafar, "Optimal use of current and outdated channel information: Degrees of freedom of the MISO BC with mixed CSIT," IEEE Commun. Lett., vol. 16, pp. 1084-1087, Jul. 2012.

[13] M. Maddah-Ali, A. Motahari, and A. K. Khandani, "Communication over MIMO X channels: interference alignment, decomposition, and performance analysis," IEEE Trans. Inform. Theory, vol. 54, no. 8, pp. 3457-3470, Aug. 2008.

[14] V. R. Cadambe and S. A. Jafar, "Interference aignment and degrees of freedom of the K-user interference channel," IEEE Trans. Inform. Theory, vol. 54, no. 8, pp. 3425-3441, Aug. 2008.

[15] _ - "Interference alignment and the degrees of freedom of wireless X networks," IEEE Trans. Inform. Theory, vol. 55, no. 9, pp. 3893 3908, Sep. 2009.

[16] S. Berger, T. Unger, M. Kuhn, A. Klein, and A. Wittneben, "Recent advances in amplify-and-forward two-hop relaying," IEEE Commun. Mag., vol. 47, no. 7, pp. 50-56, Jul. 2009.

[17] S. Mohajer, S. N. Diggavi, C. Fragouli, and D. Tse, "Approximate capacity of a class of Gaussian interference-relay networks," IEEE Trans. Inform. Theory, vol. 57, no. 5, pp. 2837-2864, May 2011.

[18] K. Gomadam and S. Jafar, "The effect of noise correlation in amplifyand-forward relay networks," IEEE Trans. Inform. Theory, vol. 55, no. 2, pp. 731-735, Feb. 2009.

[19] B. Rankov and A. Wittneben, "Spectral efficient protocols for halfduplex fading relay channels," IEEE J. Sel. Areas Commun., vol. 25 , pp. 379-389, Feb. 2007.

[20] T. Gou, S. A. Jafar, S. W. Jeon, and S. Y. Chung, "Aligned interference neutralization and the degrees of freedom of the $2 \times 2 \times 2$ interference channel," IEEE Trans. Inform. Theory, vol. 58, pp. 4381-4395, Jul. 2012.

[21] S.-W. Jeon, S.-Y. Chung, and S. A. Jafar, "Degrees of freedom region of a class of multisource Gaussian relay networks," IEEE Trans. Inform. Theory, vol. 57, pp. 3032-3044, May 2011.

[22] I. Shomorony and A. S. Avestimehr, "Two-unicast wireless networks: characterizing the degrees of freedom," IEEE Trans. Inform. Theory, vol. 59, pp. 353-383, Jan. 2013.

[23] C. Wang, T. Gou, and S. A. Jafar, "Multiple unicast capacity of 2-source 2-sink networks," in Proc. IEEE Global Telecommun. Conf., Houston, Dec. 2011.

[24] N. Lee and C. Wang, "Aligned interference neutralization and the degrees of freedom of the two-user wireless networks with an instantaneous relay," IEEE Trans. Commun., vol. 61, no. 9, pp. 3611-3619, Sep. 2013.

[25] V. Cadambe and S. A. Jafar, "Degrees of freedom of wireless networks with relays, feedback, cooperation and full duplex operation," IEEE Trans. Inform. Theory, vol. 55, no. 5, pp. 2334-2344, May 2009.

[26] K. Gomadam and S. A. Jafar, "Duality of MIMO multiple access channel and broadcast channel with amplity-and forward relays," IEEE Trans. Commun., vol. 58, no. 1, pp. 211-217, Jan. 2010.

[27] Z. Wang, M. Xiao, C. Wang, and M. Skoglund, "Degrees of freedom of relay-aided MIMO broadcast network with delayed CSIT," IEEE Wireless Commun. Lett., vol. 2, pp. 207-210, Apr. 2013.

[28] S. A. Jafar and S. Shamai, "Degrees of freedom region for MIMO X channel," IEEE Trans. Inform. Theory, vol. 54, no. 1, pp. 171-170, Jan 2008.

[29] T. M. Cover and J. A. Thomas, Elements of Information Theory. John Wiley \& Sons., 2006.

[30] R. J. Muirhead, Aspect of Multivariate Statistical Theory. John Wiley \& Sons., 1982. 